\title{
THE PALOMAR TESTBED INTERFEROMETER CALIBRATOR CATALOG
}

\author{
G. T. VAN BELLE \\ Michelson Science Center, California Institute of Technology, Pasadena, CA 91125; gerard@ipac.caltech.edu \\ G. VAN BELLE \\ Department of Biostatistics, University of Washington, Seattle, WA 98195-7232; vanbelle@u.washington.edu
}

M. J. CReech-EaKman

New Mexico Institute of Mining and Technology, Socorro, NM 87801; mce@kestrel.nmt.edu

J. COYNE

University of Cambridge, Cambridge, UK; jc466@cam.ac.uk

A. F. Boden, R. L. Akeson, D. R. Ciardi, K. M. Rykoski, and R. R. Thompson

Michelson Science Center, California Institute of Technology, Pasadena, CA 91125; bode@ipac.caltech.edu, akeson@ipac.caltech.edu, ciardi@ipac.caltech.edu,kmr@ipac.caltech.edu, thompson@ipac.caltech.edu

\author{
B. F. LANE \\ Massachusetts Institute of Technology; blane@mit.edu \\ AND \\ The PTI Collaboration \\ Jet Propulsion Laboratory and Michelson Science Center \\ Received 2007 April 9; accepted 2007 November 15
}

\begin{abstract}
The Palomar Testbed Interferometer (PTI) archive of observations between 1998 and 2005 is examined for objects appropriate for calibration of optical long-baseline interferometer observations - stars that are predictably pointlike and single. Approximately 1400 nights of data on 1800 objects were examined for this investigation. We compare those observations to an intensively studied object that is a suitable calibrator, HD 217014, and statistically compare each candidate calibrator to that object by computing both a Mahalanobis distance and a principal component analysis. Our hypothesis is that the frequency distribution of visibility data associated with calibrator stars differs from noncalibrator stars such as binary stars. Spectroscopic binaries resolved by PTI, objects known to be unsuitable for calibrator use, are similarly tested to establish detection limits of this approach. From this investigation, we find more than 350 observed stars suitable for use as calibrators (with an additional $\approx 140$ being rejected), corresponding to $\gtrsim 95 \%$ sky coverage for PTI. This approach is noteworthy in that it rigorously establishes calibration sources through a traceable, empirical methodology, leveraging the predictions of spectral energy distribution modeling but also verifying it with the rich body of PTI's on-sky observations.
\end{abstract}

Subject headings: binaries: general — catalogs — infrared: stars — instrumentation: high angular resolution — instrumentation: interferometers — stars: fundamental parameters — stars: imaging techniques: high angular resolution — techniques: interferometric

Online material: color figures, machine-readable tables

\section{INTRODUCTION}

Visible and near-infrared interferometers are powerful tools for measuring the minute angular sizes of nearby stars. However, establishing absolute system responses in the presence of atmospheric turbulence and instrument imperfections is a challenging proposition that requires careful attention to detail when constructing an observational approach. Use of objects predicted to be pointlike calibration sources is routinely employed in astronomical interferometry in the optical (Mozurkewich et al. 1991; Boden et al. 1998a; Bordé et al. 2002; van Belle \& van Belle 2005). However, given the incomplete nature of our knowledge of these sources, it is not enough to merely predict the expected fringe visibility from these objects - calibration sources need to be rigorously evaluated for their actual observed interferometric visibility and its appropriateness for use in calibrating the instrument. Herein we examine the body of calibration data taken on the sky from near-infrared, long-baseline interferometric measurements taken with the Palomar Testbed Interferometer
(PTI). Similar efforts have begun for the Very Large Telescope Interferometer (VLTI; Richichi \& Percheron 2005).

PTI is an $85-110 \mathrm{~m} \mathrm{H}$ - and K-band (1.6 and $2.2 \mu \mathrm{m})$ interferometer located at Palomar Observatory and is described in detail in Colavita et al. (1999). It has three $40 \mathrm{~cm}$ apertures used in pairwise combination for measurement of stellar fringe visibility on sources that range in angular size from 0.05 to 5.0 mas, being able to resolve individual sources $\theta \gtrsim 1.0$ mas in size. PTI has been in nightly operation since 1997 , with minimum downtime for throughout the intervening years. The data from PTI considered herein covers the range from the beginning of 1998 (when the standardized data pipeline went into place) until the end of 2005 (when the analysis of this manuscript was begun). Over the 8 years of operation discussed in this study, PTI was on the sky in its single-star visibility mode 1390 nights, ${ }^{1}$ producing

\footnotetext{
1 The remaining nights were spent on other instrument modes or were lost to maintenance or weather.
} 
over 83,000 125 s stellar "scans" on 1818 individual objects. PTI has a minimum K-band fringe spacing of $\approx 4.3$ mas at the sky position of the calibration objects, making many of these stars readily resolvable.

As is standard practice in optical interferometry, a typical set of observations with PTI involves observation of target objects of scientific interest, bracketed by calibration objects. The calibration objects serve the purpose of characterizing the point response, or system visibility $\left(V_{\text {sys }}^{2}\right)$, of the interferometer in conjunction with the atmosphere, and as such, the interleaved calibratortarget-calibrator observations are done on timescales expected to be shorter than the seeing evolution time of the atmosphere (typically less than 15 minutes). By measuring $V_{\text {sys }}^{2}$, a properly normalized measurement of the target star's visibility may be obtained for meaningful astrophysical interpretation (van Belle \& van Belle 2005). These calibration objects are selected a priori to be as close to pointlike as is possible, having visibilities measured by PTI to be very nearly unity. The primary limitation on selection of truly pointlike calibrators is the sensitivity of the instrument, coupled with considerations of dynamic range. Toward the end of having nearly pointlike calibrators, calibrator lists are vetted for binary systems, which are excluded due to the subunity system response measured from such systems. The decrease of the measured visibility from the desired characterization of $V_{\text {sys }}^{2}$ is due to the interferometer's ability to resolve out even milliarcsecond separation binaries.

Unfortunately, incomplete knowledge of the true physical nature of the calibration objects can result in binary systems not being properly excluded during the vetting process that selects potential calibrators. Given this reality of calibrator selection, a number of additional steps are taken to ensure rigorously defensible characterization of $V_{\text {sys. }}^{2}$. First, given the uncertain nature of possible calibrators, employing multiple calibrators for any given target star observation is also standard operating procedure for PTI observations. Second, these clusters of calibrators are observed on multiple nights, since chance geometry may make a binary system readily apparent on one night but not on another. Finally, these objects may be compared to known "good" calibrators for appropriateness as calibration objects, which this study examines in detail.

\section{APPROACH}

To establish a properly normalized squared visibility ${ }^{2}$ for a science target in the expected $[0: 1]$ range, the system response is used to account for the effect of instrumental and atmospheric imperfections and normalize the visibility measured for that target, $V_{\text {meas }}^{2}$ :

$$
V_{\text {norm }}^{2}(\text { target })=\frac{V_{\text {meas }}^{2}(\text { target })}{V_{\text {sys }}^{2}}
$$

The system visibility, $V_{\text {sys }}^{2}$, is established from measurements of pointlike calibration sources. However, due to the extreme resolution of interferometric instruments, event nominally "unresolved" objects need to have their measured $V_{\text {meas }}^{2}$ corrected for partial resolution:

$$
V_{\text {sys }}^{2}=\frac{V_{\text {meas }}^{2}(\text { calibrator })}{V_{\text {pred }}^{2}(\text { calibrator })} .
$$

\footnotetext{
2 Squared visibility is referred to herein as simply "visibility," which is consistent with other articles in the literature.
}

Interleaved observations of targets and calibrators are done on a timescale expected to be less than the atmospheric seeing evolution time, which at the Palomar site is typically 30 minutes or longer. PTI's automated star queue duty cycle is $4-8$ minutes per star, so calibrator-target-calibrator interleaving is comfortably accomplished with sufficient cadence to satisfy this requirement.

Assuming that a uniform disk brightness profile appropriately characterizes the stellar disk of the calibration source, the predicted visibility $V_{\text {pred }}^{2}$ may be derived from some expectation of the star's angular size, $\theta$, and the known system configuration parameters of baseline $B$ and wavelength, $\lambda$ :

$$
V_{\mathrm{pred}}^{2}=\left[\frac{2 J_{1}(\pi \theta B / \lambda)}{\pi \theta B / \lambda}\right]^{2}
$$

In estimating $V_{\text {pred }}^{2}$, and by extension $V_{\text {sys }}^{2}$, it is important to properly quantify errors in $B, \lambda$, and $\theta$, and propagate them through the Bessel function. The need for "pointlike" calibrators is prompted by potential unknown biases present in the a priori estimation of the calibrator angular size, $\theta$, and to avoid nonlinear effects found in the Bessel function. Proper error propagation is observed throughout all of these steps, including propagation of uncertainties in angular size estimate, $\sigma_{\theta}$, into $V_{\text {pred }}^{2}$ and measurement error, $\sigma_{V^{2}}$, for both $V_{\text {meas }}^{2}$ (target) and $V_{\text {meas }}^{2}$ (calibrator). The particulars and implications of this requirement are examined in much greater detail in van Belle \& van Belle (2005). In practice, for calibrators previously uncharacterized by PTI, two or more calibration sources are used in tandem to all for crosscalibration and elimination of bad calibrators.

For this study, the full list of stars observed by PTI from 1998 to 2005 was collected from the PTI archive. ${ }^{3}$ A set of calibrator selection criteria was established $(\S 3)$, and for each of the objects in the archive that qualified as a potential calibrator, a spectral energy distribution (SED) fit was used to refine its predicted angular size $(\S 4)$. Objects that were small enough for use as calibrators were kept as possible calibrators. The predicted angular size could then be used to account for the partial resolution by the interferometer of the object's angular size in attempting to normalize the object's raw interferometer data $(\S 5)$. The data obtained from the normalization step were then examined with Mahalanobis distance and principal component analysis tests for departures from point-source response and evidence of hidden binarity ( $\S$ 6). Finally, we examine the likelihood that stars observed by PTI that appear to be solid calibrators are actually undetected binaries $(\S 7)$.

\section{CALIBRATOR SELECTION CRITERIA}

In order to maximize the likelihood that any random star observable by PTI is appropriate for use as a calibrator, a number of criteria have been developed over the years of operation of the instrument. Since each principal investigator selected his or her own calibration sources for their projects, it is unclear the exact criteria that went into the selection of both targets and calibrators found in the complete PTI archive. However, since we are evaluating the sources after the fact, we may vet the observed list of potential calibrators using a single homogenous set of criteria:

1. Potential calibration sources must be bright enough to be tracked by PTI in the appropriate available dynamic range. This translates roughly to a $V$-band magnitude of $\sim 10.0$ or brighter (for tip-tilt tracking), and a $K$-band magnitude of $\sim 5.0$ or brighter (for fringe tracking).

\footnotetext{
3 Online at the Michelson Science Center, http://msc.caltech.edu.
} 
2. These sources need to be appropriately "pointlike" in size, which conservatively for PTI is $\lesssim 1.0$ mas in size, as discussed extensively in van Belle \& van Belle (2005) with final predicted size from detailed SED fitting (see $\S 4$ ). This corresponds to a measured visibility $V^{2} \gtrsim 0.90$ that serves well to characterize the system visibility (interferometer plus atmosphere) and is statistically tolerant to errors and even biases in the a priori estimation of the calibrator size.

3. Finally, for system visibility characterization, these stars must be expected to exhibit constant measured visibilities. As such, they should not be known to be a binary system, or suspected to be one, according to the astrometric references in the Hipparcos database (Perryman et al. 1997). The Hipparcos H59 multiplicity flag is of particular utility here, not only calling out those objects for which a full astrometric solution has been obtained, but those that have unexplained degrees of astrometric variability.

The 499 potential sources in the PTI database that satisfy these criteria are found in Table 1, the contents of which are discussed in the next section. The PTI data available for these sources will then be evaluated in $\S 6$ for evidence of binarity or departures in a priori size expectations that make them unsuitable for use as calibrators.

\section{SPECTRAL ENERGY DISTRIBUTION FITTING}

For each of the potential calibrator stars observed in this investigation, a spectral energy distribution (SED) fit was performed. This fit was accomplished using photometry available in the literature as the input values, with template spectra appropriate for the spectral types indicated for the stars in question. The template spectra, from Pickles (1998), were adjusted by the fitting routine to account for overall flux level, wavelength-dependent reddening, and expected angular size. Reddening corrections were based on the empirical reddening determination described by Cardelli et al. (1989), which differs little from van de Hulst's theoretical reddening curve number 15 (Johnson 1968; Dyck et al. 1996). Both narrowband and wideband photometry in $0.3-30 \mu \mathrm{m}$ were used as available, including Johnson $U B V$ (see, e.g., Eggen 1963, 1972; Moreno 1971), Stromgren $u b v y \beta$ (Piirola 1976), 2MASS $J K_{s},{ }^{4}$ other JHK (Gezari et al. 1996), Geneva (Rufener 1976), Vilnius UPXYZS (Zdanavicius et al. 1972), and WBVR (Kornilov et al. 1991); flux calibrations were based on the values given in Cox (2000). For each star, the primary references for the photometric data are given in Table 1.

Starting with a reference spectral type and luminosity class as cited by SIMBAD, template spectra were fit to the photometric data. Templates in adjacent locations in spectral type and luminosity class were also tested for best fit, with the fit with best $\chi^{2}$ being selected in the end for use in this study. For example, a star indicated by SIMBAD to be a G2 IV would have its photometry fit to the nine templates of spectral type G1, G2, and G3, and for luminosity classes V, IV, and III. From the best SED fit, estimates were obtained for each star for their bolometric flux $\left(F_{\mathrm{BOL}}\right)$, angular size $\left(\theta_{\mathrm{EST}}\right)$, and reddening $\left(A_{V}\right)$; effective temperature was fixed for each of the Pickles (1998) library spectra. The results of the fitting are given in Table 2, and an example SED fitting plot is given in Figure 1.

Also given in Table 2 are estimates of luminosity for each of these stars. The first estimate was derived from the bolometric flux from the fitting, combined with a Hipparcos distance (Perryman et al. 1997); a small number of these objects $(N=11)$ did not

\footnotetext{
${ }^{4}$ Vizier Online Data Catalog, 2246 (R. M. Cutri et al., 2003)
}

have such data available and a distance was estimated from comparison of the apparent visible brightness $m_{V}$ to the visible $M_{V}$ brightness expected from the best-fit spectral type (Cox 2000), and the SED fit reddening. The second estimate was established from the luminosity expected from the best-fit spectral type. The agreement between these two disparate estimates provided us with additional confidence in our fits, particularly from the standpoint of selection of proper luminosity class for the SED fit.

For our calibration sources, the a priori estimate of their angular size $\theta_{\mathrm{EST}}$ is necessary to account for residual resolution that may be afforded by an interferometer's extraordinarily long baselines. With an expected limb darkened size of $\theta_{\mathrm{EST}} \leq 1.00 \mathrm{mas}$ from the SED fit, calibrators have predicted $V_{\text {pred }}^{2}$ values of $>92 \%$ for a $85 \mathrm{~m}$ baseline used at $2.2 \mu \mathrm{m}$, and $>86 \%$ for a $110 \mathrm{~m}$ baseline. We consider this size effectively identical to its uniform disk size, since for most of our potential calibration sources, their effective temperatures are in excess of $\sim 5000 \mathrm{~K}$, and the difference between the uniform disk and limb darkened sizes is at the few percent level (Davis et al. 2000; Claret \& Hauschildt 2003), which is far less than our size estimate error. Ideally, a calibration source would be sufficiently pointlike that its measured visibility $V_{\text {meas }}^{2}$ would be indistinguishable from unity, but unfortunately the current system sensitivity does not afford that option. The uncertainty in the calibrator visibility represents one of the fundamental limitations of the system visibility accuracy (van Belle $\&$ van Belle 2005). However, our selection of calibrators has been carefully made to be sufficiently small $(\leq 1.00$ mas) in diameter such that there are no concerns about a varying system calibration due to unaccounted-for calibrator surface morphology, and such that a $\leq 5 \%$ uncertainty in angular size will translate to less than a $\leq 1 \%$ uncertainty in its predicted visibility $V_{\text {pred }}^{2}$ for PTI.

\section{OBTAINING NORMALIZED PTI OBSERVATIONS}

PTI generates its $V_{\text {meas }}^{2}$ observables through pairwise combination of the starlight collected by two siderostats through a 50/ 50 beam combiner optic; the combined starlight beams that result are fed into a NICMOS3 detector dewar. The PTI beamtrain from the siderostats to the beam combiner includes path length compensation that equalizes the path for each telescope from the star to the beam splitter to $\sim 20 \mathrm{~nm}$. Temporally dithering the path length compensation within one atmospheric coherence time a distance of one wavelength about this "white light" fringe position results in maximum constructive and destructive interference between the two starlight beams. Measurement of this temporally modulated photometric signal leads to a characterization of the $V_{\text {meas }}^{2}$. Discussion of the PTI fringe detection and tracking particulars is given in significantly greater detail in Colavita et al. (1999).

The $V_{\text {meas }}^{2}$ observables used in any typical PTI study are the synthetic wideband $V_{\text {meas }}^{2}$ values, given by an incoherent signalto-noise (SNR) weighted average of the individual five narrowband channel $V_{\text {meas }}^{2}(\lambda)$ values in the PTI spectrometer (Colavita 1999). In a similar fashion, incoherent SNR-weighted average bandpasses $\lambda$ were determined from the raw data. The PTI H and $\mathrm{K}$ wave bands are excellent matches to the CIT photometric system (Elias et al. 1982,1983). Separate calibrations and fits to the narrowband and synthetic wideband $V_{\text {meas }}^{2}$ data sets yield statistically consistent results, with the synthetic wideband data exhibiting superior SNR. Since there is no need in this study for independent narrowband values, we consequently consider only the synthetic wideband data.

The stars examined in this study were observed by PTI at 1.6 and $2.2 \mu \mathrm{m}$ on 1390 observing nights between 1998 January 1 


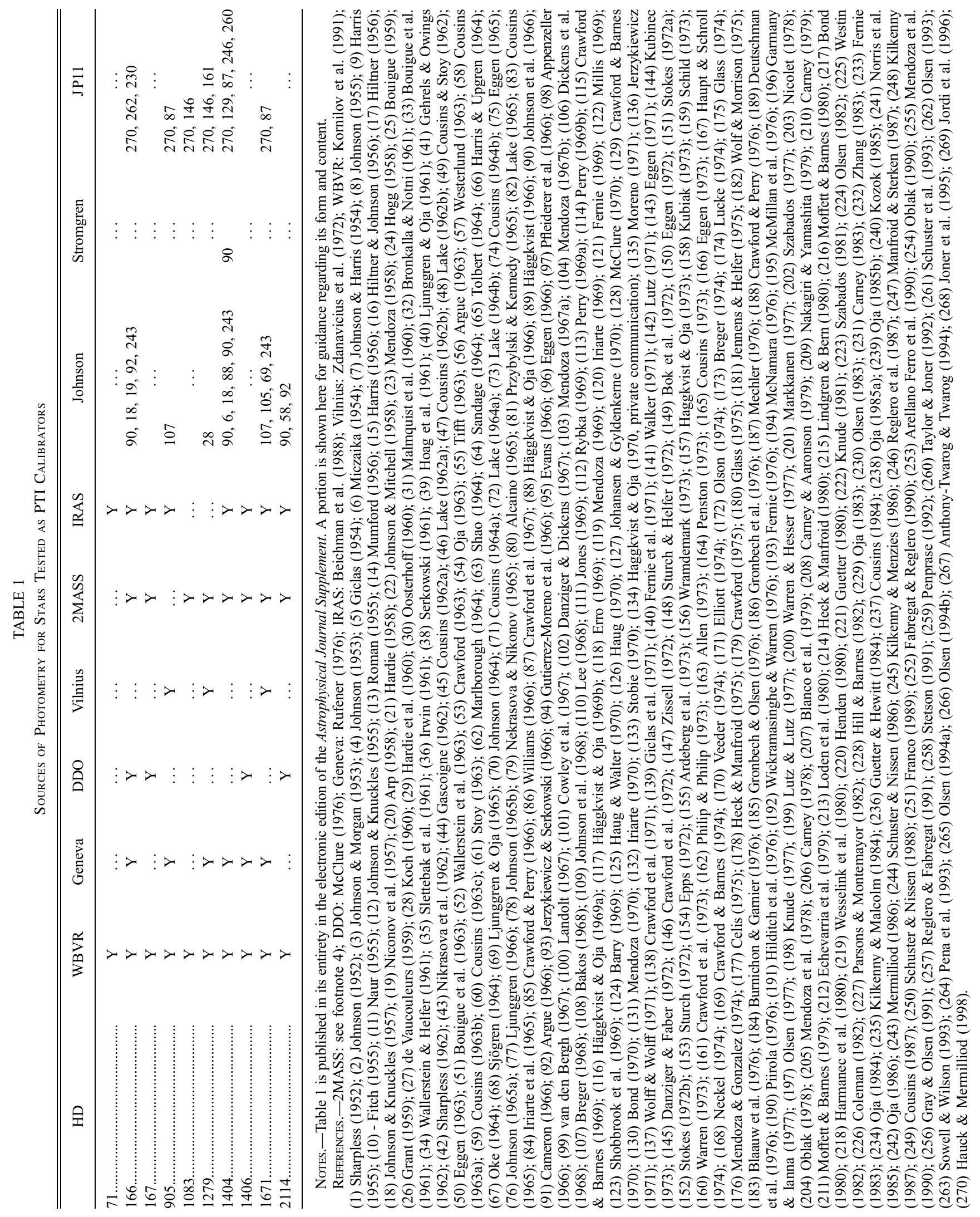


TABLE 2

Summary of Sed fits for Potential Pti Calibrator Stars, Including Total Number of Photometric Points Used, $N_{\text {Phot }}$, Reduced Chi-Squared $\chi_{\nu}^{2}$, Reddening $A_{V}$, Bolometric Flux at the Source $F_{\text {Bol }}$, Luminosity Estimate from Bolometric Flux $L_{\mathrm{Fbol}}$ and from Spectral Type $L_{\mathrm{SP}}$, and Estimated Angular Size $\theta_{\mathrm{EST}}$

\begin{tabular}{|c|c|c|c|c|c|c|c|c|}
\hline HD & $\begin{array}{l}\text { Spectral } \\
\text { Type }\end{array}$ & $N_{\text {РНОT }}$ & $\chi_{\nu}^{2}$ & $\begin{array}{c}A_{V} \\
(\mathrm{mag})\end{array}$ & $\begin{array}{c}F_{\mathrm{BOL}} \\
\left(10^{8} \mathrm{ergs} \mathrm{cm}^{-2} \mathrm{~s}^{-1}\right)\end{array}$ & $\begin{array}{l}\log L_{F_{\mathrm{BOL}}} \\
\quad\left(L_{\odot}\right)\end{array}$ & $\begin{array}{c}\log L_{\mathrm{SP}} \\
\left(L_{\odot}\right)\end{array}$ & $\begin{array}{l}\theta_{\mathrm{EST}} \\
(\mathrm{mas})\end{array}$ \\
\hline 71............................. & K1 III & 3 & 4.39 & $0.38 \pm 0.07$ & $7.45 \pm 0.17$ & $2.11 \pm 0.11$ & $1.88 \pm 0.50$ & $0.690 \pm 0.036$ \\
\hline 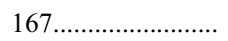 & G8 III & 8 & 0.76 & $0.13 \pm 0.03$ & $7.34 \pm 0.09$ & $1.94 \pm 0.50$ & $1.74 \pm 0.50$ & $0.594 \pm 0.036$ \\
\hline $905 \ldots \ldots \ldots \ldots$ & F0 V & 42 & 0.85 & $0.09 \pm 0.01$ & $14.40 \pm 0.13$ & $0.74 \pm 0.02$ & $0.85 \pm 0.25$ & $0.400 \pm 0.010$ \\
\hline $1083 \ldots \ldots \ldots \ldots \ldots \ldots \ldots$ & A0 IV & 16 & 0.44 & $0.03 \pm 0.02$ & $8.54 \pm 0.14$ & $1.63 \pm 0.34$ & $2.46 \pm 0.33$ & $0.167 \pm 0.010$ \\
\hline 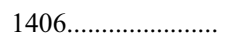 & K2 III & 15 & 1.30 & $0.23 \pm 0.02$ & $6.87 \pm 0.08$ & $1.74 \pm 0.46$ & $1.94 \pm 0.50$ & $0.728 \pm 0.037$ \\
\hline 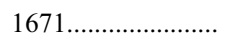 & F5 IV & 41 & 0.33 & $0.12 \pm 0.02$ & $26.75 \pm 0.23$ & $1.30 \pm 0.12$ & $1.55 \pm 0.33$ & $0.626 \pm 0.030$ \\
\hline 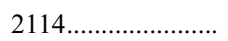 & G5 III & 17 & 0.72 & $0.00 \pm 0.05$ & $16.21 \pm 0.20$ & $2.22 \pm 0.61$ & $1.67 \pm 0.50$ & $0.805 \pm 0.054$ \\
\hline
\end{tabular}

Notes.-Table 2 is published in its entirety in the electronic edition of the Astrophysical Journal Supplement. A portion is shown here for guidance regarding its form and content.

${ }^{\text {a }}$ No Hipparcos distance available; distance estimated from comparison of apparent $m_{V}$ with absolute $M_{V}$ expected from the spectra type.

and 2005 December 31. For most of the nights, PTI's NS $110 \mathrm{~m}$ baseline (37.1 $\mathrm{m} \mathrm{E}, 103.3 \mathrm{~m} \mathrm{~N},-3.3 \mathrm{~m} \mathrm{Z}$ ) was utilized; the NW $86 \mathrm{~m}$ baseline $(-81.7 \mathrm{~m} \mathrm{E},-28.2 \mathrm{~m} \mathrm{~N}, 3.1 \mathrm{~m} \mathrm{Z})$ was used the second most, with the SW $87 \mathrm{~m}$ baseline $(-44.6 \mathrm{~m} \mathrm{E}, 75.1 \mathrm{~m} \mathrm{~N}$, $-0.2 \mathrm{~m} \mathrm{Z}$ ) being used least. The specific baselines used, in terms of number of nights and number of scans, is given in Table 3 for each star; a summary of the overall observations is given in Table 4. The potential calibration objects were observed multiple times during each of these nights, and each observation, or scan, was approximately $125 \mathrm{~s}$ long. For each scan, we computed a mean $V_{\text {meas }}^{2}$ value from the scan data, and the error in the $V_{\text {meas }}^{2}$ estimate from the rms internal scatter (Colavita et al. 1999; Colavita 1999).

\section{STATISTICAL TESTS \\ FOR THE OBSERVATIONAL DATA}

\subsection{Peg: The PTI "Gold Standard" Calibrator}

51 Peg (HD 217014, HP 113357, HR 8729) is well known throughout the astronomical community as the first solar-like

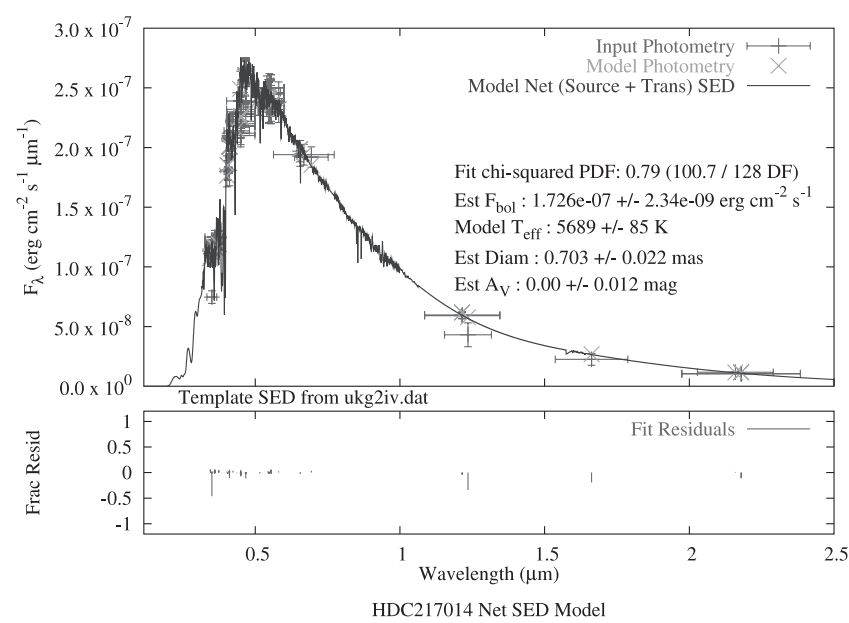

FIG. 1.- Spectral energy distribution fitting for HD 217014, as discussed in $\S 6.1$, with a G2 IV spectral template (Pickles 1998) being fit to the wide- and narrowband photometry available for the star. Vertical bars are errors associated with the photometric data; horizontal bars represent the bandwidth of each photometric data point. [See the electronic edition of the Supplement for a color version of this figure.] star for which an extrasolar planet has been detected (Mayor \& Queloz 1995; Marcy et al. 1997). When the sinusoidal radial velocity signature was detected in 1995, the $\sin i$ ambiguity in the mass term led some to suspect that perhaps what was being observed was a binary star system in a face-on orientation, rather than a star-planet pairing.

Interferometric observations have the potential to detect such binary star systems through spatially resolving and directly detecting the individual components. As such, when PTI operations began in 1996, a vigorous campaign of 51 Peg observations was begun to attempt to detect any putative stellar companion of the primary star. Despite some ambiguous initial indications associated with a new instrument learning curve, in the end a rigorous evaluation of the PTI data excluded the presence of a companion brighter than $\Delta K<4.27$ [corresponding to a brightness ratio $r(K)>0.020$ ] at the $99 \%$ confidence level, corresponding to an upper mass limit on the 51 Peg secondary of $0.22 M_{\odot}$ (Boden et al. 1998b). Given the unusually detailed evaluation of the 18 nights available (at that time) of PTI data on 51 Peg, it now constitutes a "gold standard" calibration source, as far as being an object whose nature as a system possessing a single star has been established to the limits of PTI's detection ability. For this particular investigation, we may leverage that detailed investigation of $51 \mathrm{Peg}$ to use it as a reference calibration object, to which other potential calibrators may be compared.

\subsection{Known Bad Calibrators: PTI Binaries}

In contrast to $51 \mathrm{Peg}$, we may also select well-studied PTI sources as known bad calibrators - namely, the binary stars that PTI has observed over the years. The full roster of published PTI binaries, along with $51 \mathrm{Peg}$, is summarized in Table 5. Also included on this list is an unpublished binary star, HD 178449, which with a primary-secondary brightness ratio of $\Delta K \sim 3.5-$ 3.8 represents the "worst case" binary for our various methods to attempt to detect; as discussed in more detail in $\S 7$, this level of $\Delta K$ is consistent with the median value of $V_{\text {meas }}^{2} \sim 0.93-0.95$ in equation (8).

These known binary stars are of great utility to the evaluation of our unknown potential calibrator objects. By subjecting these known binary stars to our statistical tests for appropriateness of a calibration star, we may test the sensitivity of those tests to binaries of varying brightness ratio and separation. 
TABLE 3

Summary of PTi Observations for Each Potential Calibrator, Including Nights Observed and Number of Calibrated Scans, Both Overall and By Baseline

\begin{tabular}{|c|c|c|c|c|c|c|c|c|c|}
\hline \multirow[b]{2}{*}{ HD } & \multirow[b]{2}{*}{$\begin{array}{l}\text { RAW Nights } \\
\text { OBSERVED }\end{array}$} & \multicolumn{2}{|c|}{ All Baselines } & \multicolumn{2}{|c|}{ North-South } & \multicolumn{2}{|c|}{ NoRth-West } & \multicolumn{2}{|c|}{ South-West } \\
\hline & & $\begin{array}{c}\text { Calibrated } \\
\text { Nights }\end{array}$ & $\begin{array}{l}\text { Calibrated } \\
\text { Scans }\end{array}$ & $\begin{array}{c}\text { Calibrated } \\
\text { Nights }\end{array}$ & $\begin{array}{l}\text { Calibrated } \\
\text { Scans }\end{array}$ & $\begin{array}{l}\text { Calibrated } \\
\text { Nights }\end{array}$ & $\begin{array}{c}\text { Calibrated } \\
\text { Scans }\end{array}$ & $\begin{array}{c}\text { Calibrated } \\
\text { Nights }\end{array}$ & $\begin{array}{c}\text { Calibrated } \\
\text { Scans }\end{array}$ \\
\hline $71 \ldots \ldots \ldots \ldots \ldots \ldots$ & 2 & 2 & 5 & $\ldots$ & $\ldots$ & 2 & 5 & $\ldots$ & $\ldots$ \\
\hline $166 \ldots \ldots \ldots \ldots \ldots . .$. & 67 & 67 & 270 & 53 & 217 & 12 & 49 & 2 & 4 \\
\hline $167 \ldots \ldots \ldots \ldots \ldots \ldots$ & 1 & 1 & 2 & 1 & 2 & $\ldots$ & $\ldots$ & $\ldots$ & $\ldots$ \\
\hline 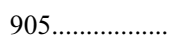 & 4 & 4 & 11 & 3 & 7 & 1 & 4 & $\ldots$ & $\ldots$ \\
\hline $1404 \ldots \ldots \ldots \ldots \ldots$ & 107 & 104 & 367 & 71 & 243 & 29 & 108 & 4 & 16 \\
\hline 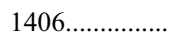 & 2 & 2 & 12 & 2 & 12 & $\ldots$ & $\ldots$ & $\ldots$ & $\ldots$ \\
\hline $1671 \ldots \ldots \ldots \ldots . . . . . .$. & 22 & 22 & 72 & 22 & 72 & $\ldots$ & $\ldots$ & $\ldots$ & $\ldots$ \\
\hline $2114 \ldots \ldots \ldots \ldots \ldots$ & 2 & 2 & 2 & 2 & 2 & $\ldots$ & $\ldots$ & $\ldots$ & $\ldots$ \\
\hline
\end{tabular}

Notes.-Table 3 is published in its entirety in the electronic edition of the Astrophysical Journal Supplement. A portion is shown here for guidance regarding its form and content.

\subsection{Moment Values for $51 \mathrm{Peg}$}

For detailed statistical testing of the visibility data on each of our potential calibrator stars found in Table 2, we sought to compare the ensemble of $51 \mathrm{Peg} V_{\text {norm }}^{2}$ points with the various sets associated each potential calibrator star first by means of a Mahalanobis distance comparison, and second through a principal component analysis. The variable of interest is an individual normalized PTI observation, $V_{\text {norm, } i}^{2}$, with associated error term $\sigma_{V^{2}, i}$. For each star we have a number of observations of $V_{\text {norm }}^{2}$ that form a frequency distribution. Our hypothesis is that the frequency distribution associated with calibrator stars differs from noncalibrator stars such as binary stars. The challenge is to characterize the frequency distribution. A very old technique is that of using Pearson curves, which are characterized by the first four moments of a distribution. A function of the raw moments of the frequency distribution of $V_{\text {norm }}^{2}$ for each star forms the input for our comparison with the first four moments of $51 \mathrm{Peg} V_{\text {norm }}^{2}$ data.

The observations were weighted by $w_{i}^{*}=1 /\left(\sigma_{V^{2}, i}\right)^{2}$. If $W=$ $\sum_{i} w_{i}$ for specific $k$ stars, and $w_{i}=w_{i}^{*} / W$, then the weighted mean, $m_{1}$, of $V_{\text {norm }, i}^{2}$ is $m_{1 k}=\sum_{i} w_{i} V_{\text {norm }, i}^{2}$. The weighted second, third, and fourth moments for this star are

$$
m_{j k}=\left[\sum_{i} w_{i}\left(V_{\text {norm }, i}^{2}-m_{1}\right)^{j}\right]^{1 / j}
$$

TABLE 4

Summary of Observations

\begin{tabular}{|c|c|c|c|c|}
\hline \multirow[b]{2}{*}{ BASELINES } & \multirow{2}{*}{$\begin{array}{r}\text { Number } \\
\text { of Stars }\end{array}$} & \multicolumn{3}{|c|}{ PROPORTION $^{\mathrm{a}}$} \\
\hline & & NS & NW & SW \\
\hline 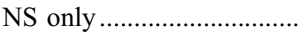 & 148 & 1.00 & $\ldots$ & $\cdots$ \\
\hline NW only $\ldots \ldots \ldots \ldots \ldots$ & 88 & $\ldots$ & 1.00 & $\ldots$ \\
\hline SW only & 1 & $\ldots$ & $\ldots$ & 1.00 \\
\hline NS, NW & 135 & 0.63 & 0.37 & $\ldots$ \\
\hline NS, SW & 5 & 0.66 & $\ldots$ & 0.34 \\
\hline NW, SW & 7 & $\ldots$ & 0.52 & 0.48 \\
\hline NS, NW, SW ....................... & 105 & 0.60 & 0.32 & 0.08 \\
\hline None (uncalibrated) ........... & 10 & $\ldots$ & $\ldots$ & $\ldots$ \\
\hline
\end{tabular}

${ }^{\text {a }}$ Distribution, on average, of the observations for each of the three PTI baselines. for $j=2,3$, and 4. For $m_{3 k}$ we retained the sign associated with the third moment. The rescaled moments have a natural interpretation; for example, $m_{2 k}$ is the standard deviation of the $V_{\text {norm }}^{2}$ values for star $k$.

These moments $m_{1 k}, m_{2 k}, m_{3 k}$, and $m_{4 k}$ form the basis for the calculation of the Mahalanobis distance and the principal component analysis distance from 51 Peg, our "gold standard." The four moment values for 51 Peg were calculated to be

$$
\begin{aligned}
& m_{1,51 \text { Peg }}=1.006659 \\
& m_{2,51 \text { Peg }}=0.041568 \\
& m_{3,51 \text { Peg }}=0.032527 \\
& m_{4,51 \text { Peg }}=0.0919526 .
\end{aligned}
$$

The moments can be used to calculate $a_{3}=$ skewness and $a_{4}=$ kurtosis statistics. For a Gaussian distribution the values are $a_{3}=0$ and $a_{4}=3$. For the $V_{\text {norm }}^{2}$ values from 51 Peg these

\begin{tabular}{|c|c|c|c|c|}
\hline Star & HD & $\begin{array}{c}a \\
\text { (mas) }\end{array}$ & $\Delta K$ & Reference \\
\hline \multirow[t]{2}{*}{64 Psc.............. } & 4676 & 6.50 & 0.11 & Boden et al. (1999b) \\
\hline & 6118 & 5.56 & 0.40 & Konacki \& Lane (2004) \\
\hline TZ Tri............. & 13480 & 2.10 & 1.75 & Koresko et al. $(1998)^{\mathrm{a}}$ \\
\hline \multirow[t]{2}{*}{ Atlas ................ } & 23850 & 12.94 & 1.86 & Pan et al. (2004) \\
\hline & 27483 & 1.26 & 0.00 & Konacki \& Lane (2004) \\
\hline o Leo ................ & 83808 & 4.46 & 1.49 & Hummel et al. (2001) \\
\hline \multirow[t]{3}{*}{12 Boo ............. } & 123999 & 3.39 & 0.61 & Boden et al. $(2000,2005)$ \\
\hline & 178449 & $\mathrm{n} / \mathrm{a}$ & $\sim 3.5-3.8$ & Unpublished \\
\hline & 195987 & 15.38 & 1.06 & Torres et al. (2002) \\
\hline$\iota \mathrm{Peg} . . . \ldots \ldots \ldots \ldots . . . .$. & 210027 & 10.33 & 1.61 & Boden et al. (1999a) \\
\hline BY Dra............. & 234677 & 4.40 & 0.57 & Boden \& Lane (2001) \\
\hline & 217014 & $\mathrm{n} / \mathrm{a}$ & $>4.27$ & Boden et al. (1998b) \\
\hline
\end{tabular}
values are

$$
\begin{gathered}
a_{3}=m_{3 k} / m_{2 k}^{3 / 2}=1.32, \\
a_{4}=m_{4 k} / m_{2 k}^{2}=12.1 .
\end{gathered}
$$

TABLE 5

Summary of Spectroscopic Binary Stars Spatially Resolved By PTi

a See discussion in $\S 6.2$. 


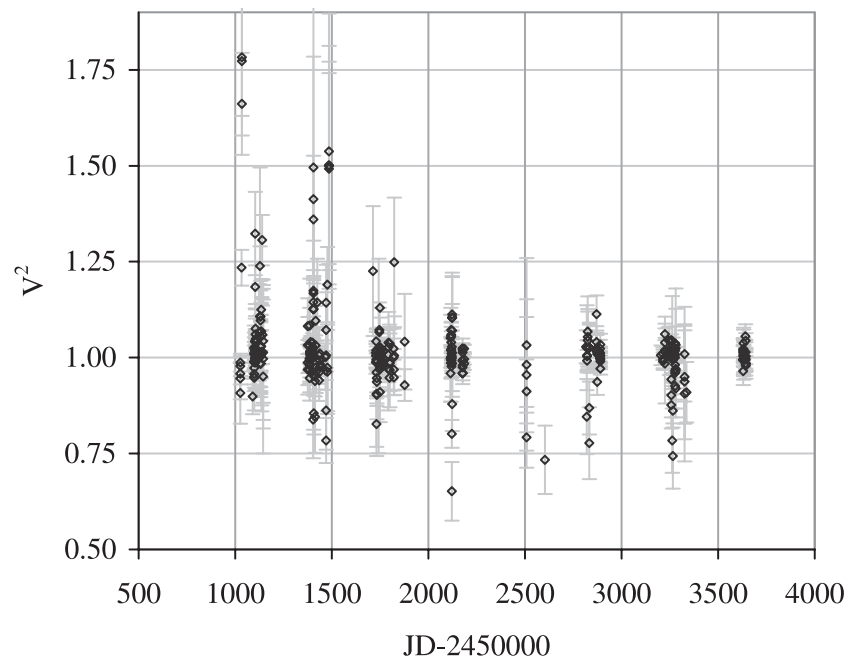

FIG. 2.- Normalized visibility measurements $\left(V_{\text {norm }}^{2}\right)$ for 51 Peg, as discussed in $\S$ 6.3. [See the electronic edition of the Supplement for a color version of this figure.]

Thus, these data suggest that the $51 \mathrm{Peg} V_{\text {norm }}^{2}$ data are skewed to the right and leptokurtic.

The skewness of the 51 Peg data is a result of there being a few, but notable, outlying data points with $V_{\text {norm }}^{2} \gg 1$, as seen in Figure 2. These data points are all associated with periods of poor system visibility when the system visibility was low, typically $V_{\text {sys }}^{2}<0.60$, which is usually a result of poor seeing; of the 340 data points in the $51 \mathrm{Peg}$ data set, 20 were in the low system visibility category. Up until this point in the analysis, all of the data from 51 Peg contributed to the statistical analysis, in order to examine all nights in a homogenous manner, regardless of atmospheric conditions. However, if we use that parameter as a guide and exclude data points with $V_{\text {sys }}^{2}<0.60$ from that analysis, we find $a_{3}=-0.048$ with $a_{4}=6.13$ - a negligible amount of skewness.

The leptokurtic nature of the $51 \mathrm{Peg} V_{\text {norm }}^{2}$ data is seen with both the full ensemble and the $V_{\text {sys }}^{2}>0.60$ subset-in terms of shape, a leptokurtic distribution has a more acute peak around the mean and longer tails. A histogram of the full 51 Peg $V_{\text {norm }}^{2}$ data set is seen in Figure 3, in bins of $\Delta V^{2}=0.05$. Two fits were compared to this binned data: the first was single Gaussian, which resulted in a mean of $\bar{V}^{2}=1.004$ and a width of 0.030 on an amplitude of 90.9, and a reduced $\chi^{2}$ per degree of freedom of $\chi^{2} /$ dof $=5.44$; the second was two Gaussians, with an identical mean but widths of 0.024 and 0.098 on amplitudes of 82.8 and 12.5 , respectively, with $\chi^{2} / \mathrm{dof}=1.32$. The second fit suggests that two sources of noise are present in the data. Restricting these fits to the $V_{\text {sys }}^{2}>0.60$ subset, we find that the single Gaussian fit has a width of 0.029 with a $\chi^{2} /$ dof $=5.02$, and the twoGaussian fit has widths of 0.080 and 0.023 with $\chi^{2} /$ dof $=1.22$; no notable change for either fit resulted in the amplitudes or $\bar{V}^{2}$ values.

These latter fits suggest that for those times when the interferometer is performing best (resulting in better $V_{\text {sys }}^{2}$ values), the wider "pedestal" Gaussian of the two-Gaussian fit reduces in width. Since the instrument is operated in a fairly uniform manner and reports lowered $V_{\text {sys }}^{2}$ values generally during periods of particularly poor atmospheric seeing, our suspicion is that the error pedestal is associated with those occasional periods of poor seeing. Such observations do not get properly normalized due to limitations of the calibration process for coping with those occasional rapid fluctuations in seeing, but are identifiable in the data

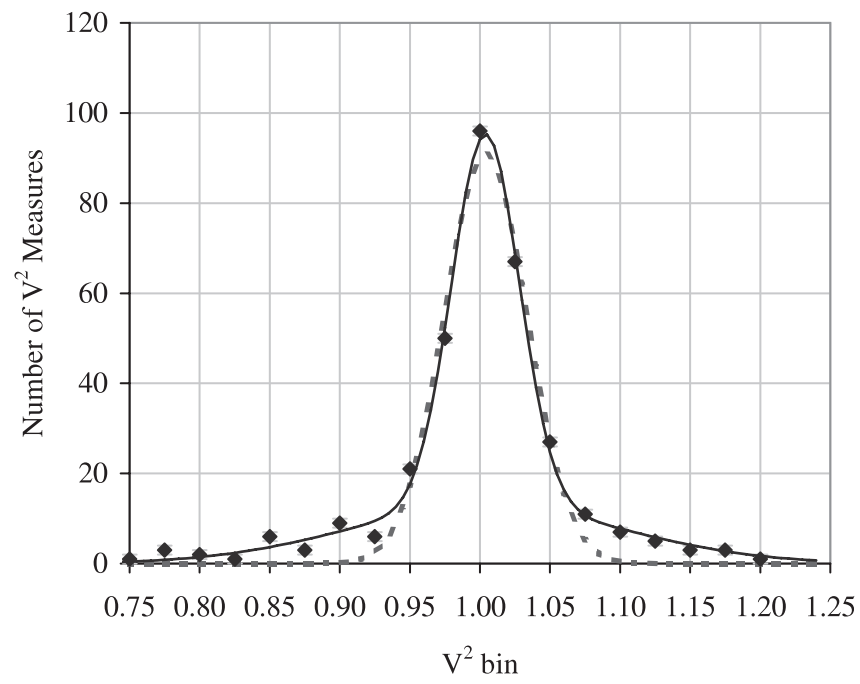

FIG. 3.- Histogram of the 51 Peg normalized visibility measurements $\left(V_{\text {norm }}^{2}\right)$ as discussed in $\S 6.3$, in bins of $\Delta V^{2}=0.05$. The dotted line is a single Gaussian fit, with amplitude of 90.9 and width 0.0297 , and the solid line is a two-Gaussian fit with amplitude/width of $82.8 / 0.0242$ and $12.5 / 0.098$, respectively. The $\chi^{2}$ per degree of freedom for the former is 5.44 , with $\chi^{2} /$ dof for the latter being 1.32. [See the electronic edition of the Supplement for a color version of this figure.]

set in part due to their low $V_{\text {sys }}^{2}$ values. Further examination of the 51 Peg data set can exclude other measurement outliers (and the spread on $V_{\text {norm }}^{2}$ will then approach the $\sim 1.5 \%$ value seen in Boden et al. 1998b) but this is time-consuming and potentially quite subjective. Thus, we maintain the integrity of the normalized visibilities being examined for all sources and do not cut for $V_{\text {sys }}^{2}$ in the following sections.

\subsection{Mahalanobis and Principal Component Analysis Distances}

The Mahalanobis distance (MD) is a multivariate generalization of one-dimensional Euclidean distance (Bartkowiak \& Jakimiec 1989; Ronen et al. 1999). Given $N$ stars characterized by $M$ variables (coordinates) $m_{j k}$, the sample distance of a particular star, $k$, from the centroid of the distances is

$$
\mathrm{MD}_{k}=\sum_{j=1}^{M}\left(\frac{m_{j k}-\overline{m_{j}}}{s_{j}}\right)^{2},
$$

where $\overline{m_{j}}$ is the mean for the $j$ th variable, and $s_{j}$ is its standard deviation. Alternatively, the MD of one star from a specific star $k^{*}$, in this case $51 \mathrm{Peg}$, say, is given by

$$
\mathrm{MD}_{k}=\sum_{j=1}^{M}\left(\frac{m_{j k}-m_{j k^{*}}}{s_{j}}\right)^{2},
$$

where $m_{j k^{*}}$ are the coordinates for star $k^{*}$. The Mahalanobis distances from 51 Peg for these stars and their ranks are listed in Table 7 below, and a histogram of the Mahalanobis distances is found in Figure 4.

Principal component analysis (PCA) is a statistical method for partitioning total variability in a sample into linear combinations that are orthogonal to each other (Ronen et al. 1999). The first principal component is generated to maximize the variation of that linear combination; the second principal component is chosen orthogonal to the first and with maximum variation conditional on 


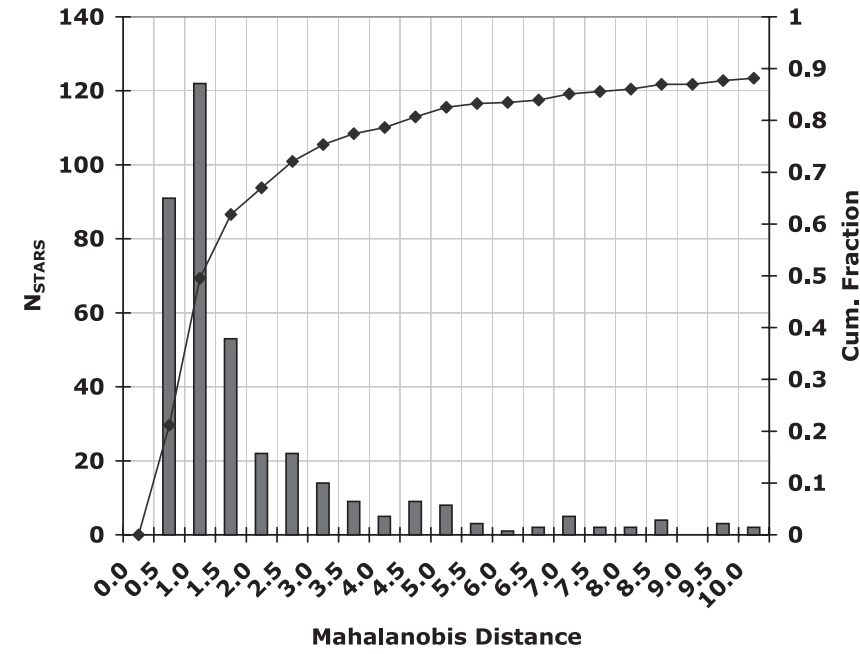

FIG. 4.-Histogram of Mahalanobis distances from HD 217014 for potential calibrator stars. [See the electronic edition of the Supplement for a color version of this figure.]

the orthogonality. For these data the four moments, $m_{1 k}, m_{2 k}, m_{3 k}$, and $m_{4 k}$, for each star $k$ again form the basis for the calculations.

The PCA is carried out on the correlation matrix, so at this stage we do not need to adjust for the variances. The principal component for 51 Peg form the basis for the distance calculation of each star from 51 Peg. Specifically, let $p_{j k}$ be the principal components for star $k$ and $p_{j k^{*}}$ the principal component for reference star $k^{*}$. Then the distance star $k$ is from the reference star is

$$
\mathrm{PD}_{k}=\sum_{j=1}^{M}\left(\frac{p_{j k}-p_{j k^{*}}}{s p_{j}}\right)^{2}
$$

where $s p_{j}$ is the standard deviation for the $j$ th principal component. The PCA distances and their ranks are given in Table 7 below, and a histogram of the PCA distances is found in Figure 5.

The choice between Mahalanobis distance and the PCA-based distance is based on convenience. The Mahalanobis distance has the advantage that it is more immediately linked to the four moments of the distribution. There is a very close correlation between the Mahalanobis distance and the PCA-based distance as seen in Figure 6 . The PCA based approach has the advantage that the contribution of each of the principal components can be calculatede.g., for the data considered here the first principal component accounts for $53 \%$ of the total variability.

We find that the first of the known PTI binaries to appear among the ranked potential calibrators of Table 6 is HD 178449not unsurprising given its large $\Delta K$ value. The large brightness ratio makes the object appear more like a calibrator than the other binary stars, and at distances of $\mathrm{MD}_{\mathrm{HD} 178449}=1.20, \mathrm{PD}_{\mathrm{HD} 178449}=$ 1.83. Consideration of the HD 178449 Maha and PCA ranks and an examination of Figures 4 and 5 provides insight into the distributions for both Maha and PCA distances. Given that the sources were preselected to likely be suitable as calibration sources, it is reasonable to expect that distributions should peak at values corresponding to suitable calibrators. If we select all stars that fall toward the smaller values of those distribution, we can have some confidence that we have statistically selected the stars that have been demonstrated in this data set to be suitable for use as calibrators. Thus, we established our cutoff at MD, PD $<1.0$. The resulting calibrator list is available online at the MSC Web site (see footnote 3).

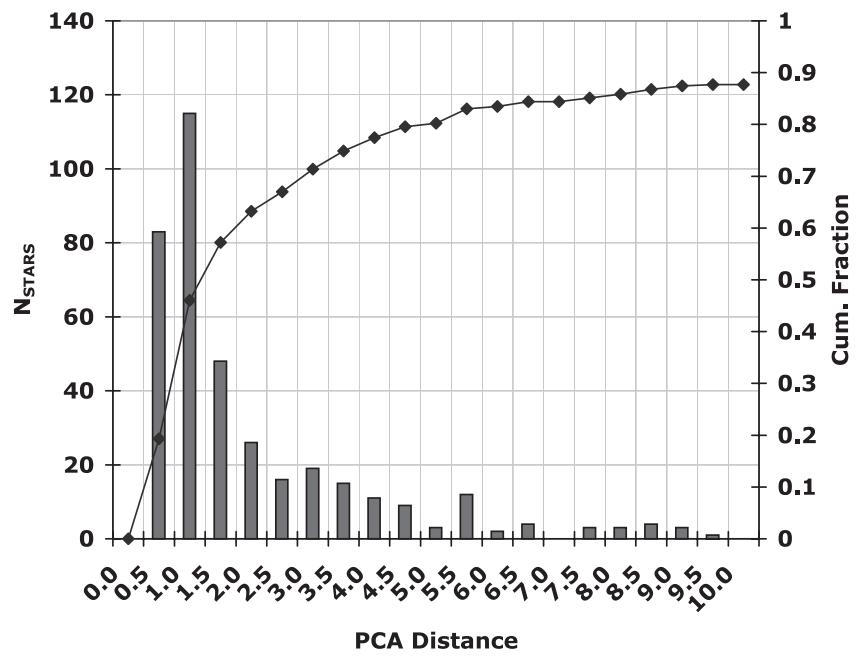

FIG. 5.- Histogram of principal component analysis distances from HD 217014 for potential calibrator stars. [See the electronic edition of the Supplement for a color version of this figure.]

It is important to note that the "rejection" of a star as a suitable calibrator from this analysis does not necessarily indicate that it is a heretofore unresolved binary - merely that, within this particular data set, the data indicate that it is unsuitable for use as a calibrator. This could be due to some previously undetected binarity, but could also be merely due to poor data quality for that particular object in the data set. A key indicator of this possibility is the fact that the stars in Table 6 that are listed as "acceptable" have, on average, a larger expected angular size ( 0.449 mas) than those in the "reject" category (0.301 mas). The smaller stars are, on average, more distant, and hence, are also on average dimmerresulting in lower signal-to-noise in the PTI system. Such objects are more susceptible to poor seeing and/or weather conditions and are ultimately more likely to be rejected due to poor data quality that is unrelated to actual system binarity. For the purposes of this

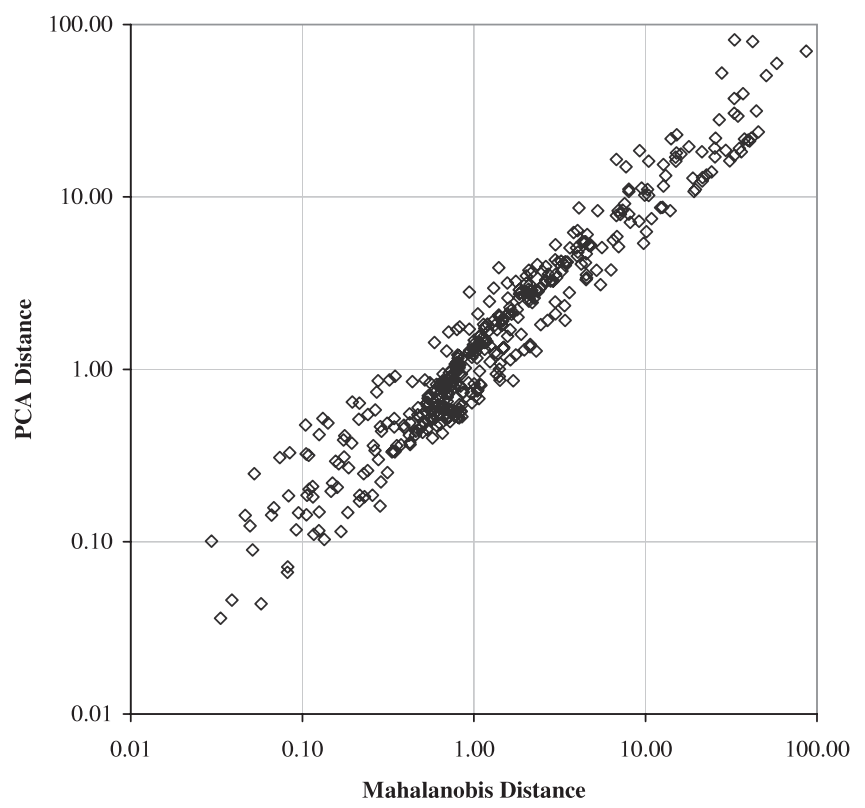

FIG. 6.-Principal component analysis vs. Mahalanobis distances from HD 217014 for potential calibrator stars. [See the electronic edition of the Supplement for a color version of this figure.] 
TABLE 6

Mahalanobis Distance Rank and Principal Component Analysis Rank of the Potential Calibrator Stars

\begin{tabular}{|c|c|c|c|c|c|c|}
\hline HD & Mahalanobis Rank & Mahalanobis Distance & PCA Rank & PCA Distance & Geometric Nondetection $^{\mathrm{a}}$ & Binary Reference \\
\hline 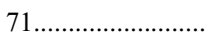 & 94 & 0.51 & 82 & 0.49 & 0.036 & $\ldots$ \\
\hline 1404 & 63 & 0.28 & 74 & 0.47 & $\ldots$ & $\ldots$ \\
\hline $1671 \ldots \ldots \ldots$ & 134 & 0.66 & 190 & 0.94 & $\ldots$ & $\ldots$ \\
\hline $2344 \ldots \ldots \ldots \ldots \ldots \ldots$ & 30 & 0.13 & 64 & 0.42 & 0.010 & $\ldots$ \\
\hline 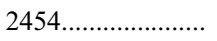 & 172 & 0.79 & 203 & 1.04 & 0.000 & $\ldots$ \\
\hline 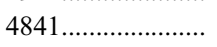 & 35 & 0.15 & 34 & 0.22 & 0.002 & $\ldots$ \\
\hline 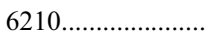 & 195 & 0.84 & 112 & 0.57 & 0.188 & $\ldots$ \\
\hline $6288 \ldots \ldots \ldots \ldots \ldots \ldots$ & 15 & 0.08 & 26 & 0.18 & 0.002 & $\ldots$ \\
\hline
\end{tabular}

Notes.-Table 6 is published in its entirety in the electronic edition of the Astrophysical Journal Supplement. A portion is shown here for guidance regarding its form and content.

${ }^{a}$ Probability of a binary system nondetection due to lack of total nights on the sky, as discussed in $\S 7$.

investigation, rejection of such sources is acceptable in exchange for attempting to eliminate false positives for acceptable calibrators.

\section{ESTIMATION OF THE UNDETECTED BINARY RATE}

One primary concern with the selection of potential calibration sources is choosing objects that are previously undetected binaries. For this reason, it is standard operating procedure to observe no fewer than two calibration sources in conjunction with astrophysical targets, allowing some means of cross-calibration between the potential calibrators. Although we cannot know for certain which of our previously unobserved calibration sources might have a heretofore undetected companion, we may attempt to characterize the likelihood with which such an undetected companion would escape detection once such a binary star system were placed on the observing queue.

We generated a synthetic, random sample of 4096 binary stars, starting with selecting an object with a random spectral type consistent with our potential calibrator list, which is within the range of $\mathrm{B} 8$ to $\mathrm{K} 1$. Luminosity class for these objects was statically assigned to be either III or V, to test the sensitivity of binary detection to primary star luminosity class. Absolute $V$ and $K$ magnitudes, radii, and masses for these primary stars were then drawn from the standard MK-type values found in Bessell \& Brett (1988) and Cox (2000). Apparent magnitude was randomly assigned a value between $m_{K}=3.5$ and 5.0, which is consistent with the apparent magnitudes of PTI calibrators. Secondary stars were then randomly generated for each of these primaries: spectral type was selected to be equal to or later than the primary star, down to a class of $\mathrm{M} 9,{ }^{5}$ and for giant star primaries, luminosity class of the secondary objects was randomly assigned either III or V. Absolute $V$ and $K$ magnitudes, radii, and masses for these secondary stars were then estimated also using the same references. Orbital separation $a$ was then randomly assigned to be between 2 and 50 Roche diameters; distance of the binary star pair was established from the distance modulus, with an apparent angular separation resulting from the distance and true separation of the primary and secondary. Periods were established from the physical separation and the masses. Orbital parameters, such as eccentricity $e$, inclination $i$, and longitude of the ascending node $\Omega$

\footnotetext{
5 Implicit in this condition is a "flat" mass ratio $q=M_{1} / M_{2}$ for binary stars, a topic that is discussed extensively in Duquennoy \& Mayor (1991) and more recently in Goldberg et al. (2003), which appears to be reasonable for this experiment.
}

were randomly assigned. Angular sizes of each component were estimated using the $V-K$ technique described in van Belle (1999); given the $m_{K}=3.5-5.0$ prior, the primaries were all well within the PTI calibrator selection criterion of $\theta<1.0$ mas. In addition, this sample well represents a potential sample of overlooked binary stars, with $a<0.1^{\prime \prime}$, regardless of luminosity class.

Our overall goal was to test this subsample for PTI's ability to detect deviations from what was expected to be normal calibrator object behavior. The expected squared visibility in a narrow passband for a binary system is given by

$$
V^{2}(\lambda)=\frac{V_{1}^{2}+V_{2}^{2} r^{2}+2 V_{1} V_{2} r \cos [(2 \pi / \lambda) \boldsymbol{B} \cdot \boldsymbol{s}]}{(1+r)^{2}},
$$

where $V_{1}$ and $V_{2}$ are the visibility moduli for the two stars alone as given by equation (3), $r$ is the apparent brightness ratio between the primary and secondary, $B$ is the projected baseline vector at the system sky position, and $s$ is the primary-secondary angular separation vector on the plane of the sky (Hummel et al. 1995; Boden et al. 1999a). $V_{1}$ and $V_{2}$ were estimated from the angular sizes estimates. A conservative detection limit would be a $3 \sigma$ threshold of $\Delta V^{2}>0.06$, roughly three times PTI's limiting $V^{2}$ precision of $\sim 0.015-0.020$, where $\Delta V^{2}$ is the difference between the expected single star $V^{2}$ as described in equation (3) and the binary star $V^{2}$ from equation (8). Objects with binary $V^{2}$ excursions less than PTI's limiting $V^{2}$ precision would effectively be single stars as far as PTI is concerned, and were excluded from consideration.

Figuring prominently into this characterization of PTI's ability to detect these synthetic binary stars is the geometric orientation of the target systems relative to the instrument's baseline in use, contained in the $\boldsymbol{B} \cdot \boldsymbol{s}$ term of equation (8). If PTI's baseline in use is orthogonal to the primary-secondary separation vector, the departure from point-source source visibility will potentially be undetectable, depending on the brightness ratio. Since the relative geometry between binary system and projected baseline varies with orbital phase, these 4096 systems were then examined over the course of 20 random epochs between the years covered by this study, 1998-2005, using PTI configurations consistent with the available baselines. As the number of nights increases, the likelihood that a binary system would remain undetected is given in Table 7 .

Two categories of binary stars were problematic for PTI: First, stars with detectable $V^{2}$ excursions $\left(\Delta V_{\max }^{2} \geq 0.06\right)$ but in poor 


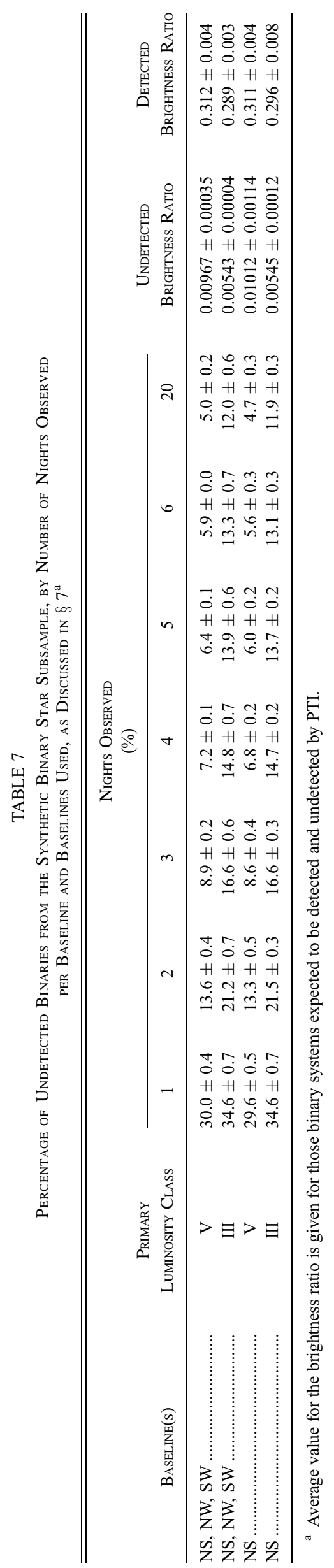


geometric orientation, due to orbital phase or apparent sky separation relative to baseline used, are found to escape initial detection by the array. However, after a few $(\sim 5)$ nights of observing, virtually all of these objects exhibited a detectable excursion and would be detected as binaries. Second, and more problematically, are the stars with $V^{2}$ excursions below the $3 \sigma$ threshold of obvious detectability, but above PTI's limiting $V^{2}$ precision of $\sim 0.015-0.020$. These objects would remain always unconfirmed as binaries, yet their varying $V^{2}$ response could affect system calibration at the $2 \%-4 \%$ level.

For example, in a single night of observing with the NS baseline, approximately $\sim 30 \%$ of the binary systems with a main sequence primary are undetected. However, this proportion drops rapidly to $\sim 5.5 \%$ after only five nights of observing, and to $\sim 5 \%$ after 20 nights of observing, as it does for using the three PTI baselines in sequence. For binary systems with giant primaries, the limiting proportion of undetected binaries is higher, at $\sim 12 \%$ after 20 nights. This larger value is consistent with a greater number of binary systems with a large brightness ratio, specifically those systems with a main sequence secondary star. This nonzero proportion of undetected binary systems is compelling motivation to employ multiple calibrators for any study with PTI, or any other interferometer. As we see in $\S 6$, we have many stars that show PTI $V^{2}$ data that are consistent with the point-response of a single, rather than binary, star system. The lingering uncertainty of undetected binarity for minimally observed stars can be drawn from the percentages given in Table 7 .

\section{DISCUSSION}

Some of the objects categorized as "acceptable" calibrators due to their PTI $V^{2}$ are still found to have references indicating possible binary nature or other astrophysical effects that may cause them to be less than suitable as calibrators; these objects are discussed below in $\S 8.1$. Interferometer observations of hierarchical systems have the potential to accidentally observe the wrong star and so appear to have a variation in angular size. Variations in metallicity will mean that a potential calibrator will not fit SED templates well for predicting size. Stars with extreme rotational velocity might exhibit angular size variations depending on interferometer baseline utilized, and "thrown off" material (as seen with the Be star phenomena) could also affect visibility measurements if the disk is detected. In addition, evidence in the literature was uncovered for rejected calibrator candidates and is discussed in $\S 8.2$.

\subsection{Objects Thought to Be Acceptable Calibrators}

Figure 7 shows an equatorial sky coverage map of statistically vetted calibrators displayed in an equal area Aitoff projection. For PTI's nominal sky accessibility of $5^{\circ}<\delta<55^{\circ}$, two or more vetted calibrators cover are available for any $10^{\circ}$ cone upon the sky for over $96 \%$ of the sky.

HD 905.- - Listed as a periodic variable star (Koen \& Eyer 2002).

HD 1279.- Shows P Cygni type profiles in UV spectra taken with IUE (Snow et al. 1994).

HD 2758.- - Listed as a binary in the Tycho-2 catalog (Fabricius et al. 2002) with a companion at position angle $69.6^{\circ}$ and separation of $0.44^{\prime \prime}$.

HD 3268.- - Listed in Cayrel de Strobel et al. (1997) as metalpoor with $[\mathrm{Fe} / \mathrm{H}]=-0.23$.

HD 15335.- - Listed in Heintz (1994) as an 0.020" astrometric binary, detected via photographic plates obtained with the $61 \mathrm{~cm}$

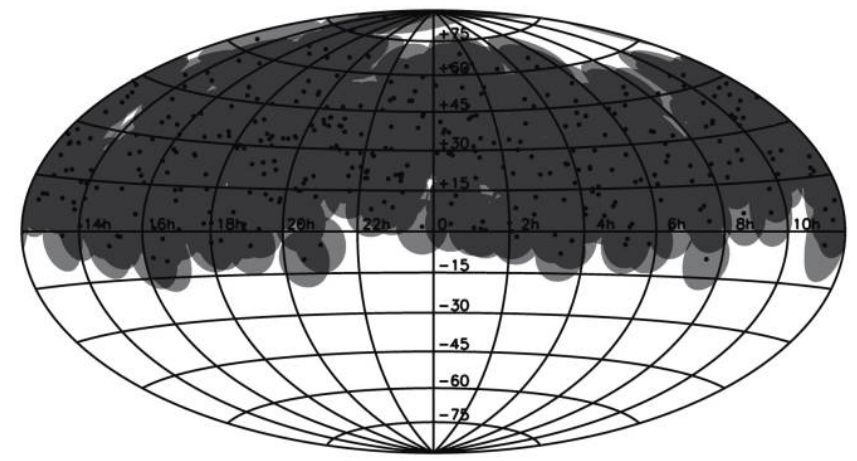

FIG. 7.-Equatorial sky coverage map of statistically vetted calibrators displayed in an equal area Aitoff projection. Light gray circles represent $10^{\circ}$ radii cones projected onto the sky, representing regions of appropriate calibrator proximity to astrophysical targets; darker gray regions represent areas with two or more calibrators within $10^{\circ}$. For PTI's nominal sky accessibility of $5^{\circ}<\delta<55^{\circ}$, these calibrators represent $\gtrsim 96 \%$ sky coverage.

Sproul refractor, although the comments cite it as "astrom., probable," and no secondary component magnitude is listed. Follow-up work by Fischer \& Valenti (2005) report a null result not only on detecting a stellar companion, but also down to planetary mass.

HD 19994 and HD 90508.-Both are indicated to be binary stars in Hale (1994). HD 19994 is notable in that it has a Jupitermass planet (Mayor et al. 2004), and the F8 V star's stellar companion is an M3 V dwarf a few arcseconds away, which makes it undetectable by PTI from a brightness ratio standpoint. Similarly, HD 90508 is a F9 V star with a M3 V companion.

HD 24357.- - Listed in Cayrel de Strobel et al. (1997) as metalrich with $[\mathrm{Fe} / \mathrm{H}]=0.30$.

HD 27524. - Listed in Cayrel de Strobel et al. (1997) as metalpoor with $[\mathrm{Fe} / \mathrm{H}]=-0.46$.

HD 28024.- - Listed in the Washington Double Star Catalog as a visual double with $104.3^{\prime \prime}$ separation and a magnitude difference of 8.23 (Worley \& Douglass 1997). It has a GCVS period of 0.148 days (Samus et al. 2004) and in addition, it is cited as a $\delta$ Scuti variable (Rodriguez et al. 2000). It also has high rotational velocity (see below).

$H D$ 28677.- - Listed in the GCVS as a possible variable star, however, no period or variability type is identified and this designation may be in doubt (Samus et al. 2004). We also note that this star is listed in a catalog of binaries detected using lunar occultation and has a separation from its companion of $16.2 \pm 5.2$ mas, at a position angle of 205.9 degrees, with a magnitude difference of $0.0 \pm 1.62 \mathrm{mag}$ at $547 \mathrm{~nm}$ (Mason 1995). There is no evidence for binarity in the PTI data, despite the nine nights of available calibrated PTI data (seven on the NS baseline, two on the NW baseline).

HD 28704. - Listed in the Washington Double Star Catalog as being a hierarchical quadruple system with separations from the primary ranging from $76.4^{\prime \prime}$ to $113.7^{\prime \prime}$ and magnitude differences with respect to the primary ranging from 0.71 to 7.3 (Worley \& Douglass 1997).

HD 31662.- - Listed in the Washington Double Star Catalog as being a visual double with separation $5.4^{\prime \prime}$ and a magnitude difference of 5.47 (Worley \& Douglass 1997). There is no evidence for binarity in the PTI data.

HD 33167.- Has a large range of metallicities in the literature, from $[\mathrm{Fe} / \mathrm{H}]=0.09$ (Cayrel de Strobel et al. 1997) to $[\mathrm{Fe} / \mathrm{H}]=$ -0.36 to -0.37 (Ibukiyama \& Arimoto 2002 and Marsakov \& Shevelev 1995, respectively). 
HD 38558.- - Noted by Adelman (2001) to have variability of $0.01 \mathrm{mag}$ in the Hipparcos data.

HD 43042.- - Listed in the Washington Double Star Catalog as being a hierarchical quadruple system with separations from the primary ranging from $32.0^{\prime \prime}$ to $91.2^{\prime \prime}$ and magnitude differences with respect to the primary ranging from $4.8^{\prime \prime}$ to $6.0^{\prime \prime}$ (Worley \& Douglass 1997).

HD 51530.-- Listed in the Washington Double Star Catalog as being a hierarchical triple system with separations from the primary of $0.5^{\prime \prime}$ and $28.2^{\prime \prime}$ and a magnitude difference of the larger separated component of 6.0 mag (Worley \& Douglass 1997). There is no evidence for binarity in the PTI data.

HD 58551.- Noted to be extremely metal-poor, with $[\mathrm{Fe} / \mathrm{H}]=$ -0.6 (Bartkevicius 1984; Cayrel de Strobel et al. 1997).

HD 58715.- Noted as a double in the Hipparcos Input Catalogue with unknown separation and magnitude difference (Turon et al. 1993). It is listed as an eruptive $\gamma$ Cas variable by (Samus et al. 2004) and has high rotational velocity (see below).

HD 58946.- - Listed as a hierarchical quadruple system spread over $5^{\prime}$. The closest companion to the primary is separated by $3.4^{\prime \prime}$ with a magnitude difference of 8.4 (Turon et al. 1993). Worley \& Douglass (1997) goes on to list the A component in the system as a spectroscopic binary. However, Galland et al. (2005) use the system as a constant RV source to demonstrate the stability of their ELODIE instrument in searching for brown dwarfs and extrasolar planets. Samus et al. (2004) lists it in the GCVS with unknown variability type. And finally Adelman (2001) shows it to have 0.01 mag of variability, as measured by Hipparcos.

HD 71148.- - Listed in the GCVS (Samus et al. 2004) as a previously misidentified variable star. It is metal-poor with $[\mathrm{Fe} / \mathrm{H}]=$ -0.15 (Ibukiyama \& Arimoto 2002).

$H D$ 75332.- Has been studied extensively in radial velocity surveys searching for extrasolar planets, however, no evidence for any are found to date (Fischer \& Valenti 2005).

$H D$ 75732. - A visual double with a separation of 85.0" and a magnitude difference of 7.2. It has been found to have planets (Fischer \& Valenti 2005), which are well below the sensitivity threshold of PTI to detect.

HD 89125.-A visual double with a separation of 7.4" (Turon et al. 1993). In addition, it is seen to have low metallicity ranging from $[\mathrm{Fe} / \mathrm{H}]=-0.19$ to -0.39 (Cayrel de Strobel et al. 1997; Marsakov \& Shevelev 1988).

$H D$ 97334.-A hierarchical quadruple system with separations from the primary ranging from $88.2^{\prime \prime}$ to $128.4^{\prime \prime}$ and magnitude differences from 1.5 to 6.0 (Worley \& Douglass 1997).

$H D$ 97633.-Flagged as a variable star by Samus et al. (2004) with the variable type and period unidentified. Further, it is extremely metal-rich, with $[\mathrm{Fe} / \mathrm{H}]=0.40$ (Cayrel de Strobel et al. 1997).

HD 103095.-Flagged as a suspected variable star by Samus et al. (2004), with the variable type and period unidentified. It has also been studied by Latham et al. (2002) for binarity and was found to have radial velocity measurements that varied at the $2-$ $3 \mathrm{~km} \mathrm{~s}^{-1}$ level, with no indication of binarity or an orbital solution.

HD 119550.- - Has also been studied by Latham et al. (2002) for binarity and was found to have radial velocity measurements that varied at the $1 \mathrm{~km} \mathrm{~s}^{-1}$ level, with no indication of binarity or an orbital solution.

$H D$ 128167.-Flagged as a suspected variable star by Samus et al. (2004) with the variable type, based on a reference, potentially a $\sigma$ Bootes variable with an unidentified period. It is identified in the Washington Double Star catalog as a member of a hierarchical triple system with separations from the primary of $248.0^{\prime \prime}$ and 237.0" and 5.34 and $6.80 \mathrm{mag}$, respectively (Worley
\& Douglass 1997). Finally, it displays a wide range of low metallicities, from $[\mathrm{Fe} / \mathrm{H}]=-0.18$ to -0.60 (Cayrel de Strobel et al. 1997).

HD 132254 and HD 134083.-Both are flagged by Nordström et al. (2004) as spectroscopic binaries, although no other supporting evidence in the literature could be found for this conclusion for either star. Indeed, in Galland et al. (2005), HD 134083 is cited as having a constant radial velocity at the $\sim 50 \mathrm{~m} \mathrm{~s}^{-1}$ level, which would preclude any binary detectable by PTI. HD 132254 was well-behaved enough for Kharchenko et al. (2004) to list the object as a candidate RV standard.

HD 136118.- - Has been studied extensively with radial velocity techniques and is know to have a planet (Santos et al. 2003, 2004), which are well below the sensitivity threshold of PTI to detect.

$H D$ 141187.- Studied in a catalog of astrometric binaries, identified using Hipparcos and Tycho data, to have nonlinear proper motion (Makarov \& Kaplan 2005). It is also seen to have high rotational velocity (see below).

HD 154345.--Listed in Samus et al. (2004) as a suspected variable star in the GCVS, however no period of variability type is listed.

HD 166205.-Flagged in Mason et al. (1999) as a suspected binary on the basis of ground-based and Hipparcos data. However, the binarity was not able to be confirmed, so it is listed in their Table 7 as a "problem star." Finally, it is flagged in Adelman (2001) as a variable star in the Hipparcos survey with $0.01 \mathrm{mag}$ of variability.

HD 171834. - Flagged as a binary with $0.1^{\prime \prime}$ separation and no notation of the secondary's magnitude in Worley \& Douglass (1997). It is also seen to have a low metallicity compared to solar of $[\mathrm{Fe} / \mathrm{H}]=-0.42$ (Cayrel de Strobel et al. 1997).

$H D$ 182488. - Has been studied extensively using the radial velocity technique and is found to have no detectable planet or stellar companions Fischer \& Valenti (2005).

$H D$ 186568. - A member of a hierarchical triple with separations of $15.4^{\prime \prime}$ and $33.9^{\prime \prime}$ and magnitude differences of 7.75 and 5.44, respectively (Worley \& Douglass 1997).

HD 187691.-A member of a hierarchical quintuple system with separations from the primary ranging from $20.5^{\prime \prime}$ to $89.7^{\prime \prime}$ and magnitude differences of 7.69-8.59 Worley \& Douglass (1997). It was used by Mazeh et al. (1996) as an RV standard in the study of orbits of three spectroscopic binary systems. Fischer \& Valenti (2005) have studied it extensively in their radial velocity searches for planets and find no evidence for a companion. And finally, it is flagged in the GCVS as a rotating variable star with no period listed (Samus et al. 2004).

$H D$ 187923. - A member of a visual double system with a separation from the secondary of $93.9^{\prime \prime}$ and a magnitude difference of 5.57 (Worley \& Douglass 1997). It has been studied extensively using radial velocity techniques and is found to have no companions Fischer \& Valenti (2005). It is flagged in the GCVS as variable, but no variability type or period is identified (Samus et al. 2004).

HD 195564.-A member of a hierarchical triple system with separations of $4.6^{\prime \prime}$ and 100.2" and magnitude differences of 5.5 and 4.2, respectively (Worley \& Douglass 1997). It has been studied extensively using radial velocity techniques and is found to have no companions Fischer \& Valenti (2005).

HD 197076.-A member of a hierarchical triple system with separations of $98.8^{\prime \prime}$ and $125.0^{\prime \prime}$ and magnitude differences of 5.45 and 6.95, respectively (Worley \& Douglass 1997). It has been studied extensively using radial velocity techniques and is found to have no companions (Fischer \& Valenti 2005). 
HD 198478. - A member of a visual double system with a separation of $20.5^{\prime \prime}$ and a magnitude difference of 5.32 (Worley \& Douglass 1997). It is flagged in the GCVS as being a pulsating variable star (Samus et al. 2004) and has an identified period of 0.20472 days (Koen \& Eyer 2002).

$H D$ 210855.-A member of hierarchical triple system with separations of $79.6^{\prime \prime}$ and $56.0^{\prime \prime}$ and magnitude differences of 5.26 and 8.46, respectively (Worley \& Douglass 1997).

HD 214680.- A member of a visual double system with a separation of $60.4^{\prime \prime}$ and a magnitude difference of 5.14 (Worley $\&$ Douglass 1997). Further, it is flagged in the GCVS as a $\beta$ Cephei variable star (Samus et al. 2004).

HD 218396.-Flagged in the GCVS as a rotating ellipsoidal variable (Samus et al. 2004) and as a probable $\lambda$ Bootis variable by Gerbaldi et al. (2003).

HD 219080.-Flagged as a new periodic variable star by Koen \& Eyer (2002).

HD 222439. - A member of a hierarchical triple system with separations of $46.6^{\prime \prime}$ and 103.2" and magnitude differences of 7.16 for both companions ( Worley \& Douglass 1997). Further, it is flagged in the GCVS as a variable star of unknown type (Samus et al. 2004).

In addition, the following stars have rotational velocities $\left(v \sin i\right.$ ) between 100 and $200 \mathrm{~km} \mathrm{~s}^{-1}$ : HD 7804, HD 18411, HD 20150, HD 26605, HD 27397, HD 27946, HD 28677, HD 30739, HD 32301, HD 32630, HD 37147, HD 50019, HD 56537, HD 60111, HD 67006, HD 79439, HD 83287, HD 85795, HD 87696, HD 92825, HD 102124, HD 107904, HD 108765, HD 110411, HD 111604, HD 116831, HD 122408, HD 125161, HD 125162, HD 132052, HD 141003, HD 143894, HD 147547 , HD 152614, HD 161868, HD 166014, HD 166205, HD 168914, HD 169702, HD 177196, HD 177756, HD 192425, HD 204153, HD 206043, HD 210129, HD 213558, HD 214734, HD 214923, HD 216735, and HD 222439. The following stars have rotational velocities higher than $200 \mathrm{~km} \mathrm{~s}^{-1}$ : HD 11946, HD 17573, HD 20418, HD 21686, HD 23630, HD 23862, HD 28024 , HD 30823, HD 58715, HD 73262, HD 93702, HD 98058, and HD 130109. These data were taken mainly from VizieR catalogs associated with Glebocki et al. (2000), Royer et al. (2002), and de Medeiros et al. (2002). Rapidly rotating stars have an additional uncertainty in their predicted angular size due to rotational distortion, which is on the order of $\sim 10 \%-15 \%$ (van Belle et al. 2006). However, if this was a significant factor, the statistical vetting of $\S 6$ would have rejected these objects as valid calibrators.

\subsection{Objects Rejected as Acceptable Calibrators}

HD 8357.-Flagged by Nordström et al. (2004) as a spectroscopic binary, with a mass ratio of $q=0.841 \pm 0.009$.

HD 8799.-Found to be listed as a visual (but not physical) binary of unspecified separation in Tokovinin \& Smekhov (2002).

HD 13480.-Flagged by Nordström et al. (2004) as a spectroscopic binary, with a mass ratio of $q=1.062 \pm 0.010$.

$H D$ 16234.-Flagged by Nordström et al. (2004) as a spectroscopic binary, with a mass ratio of $q=1.039 \pm 0.041$.

HD 18012.- Examined by Lu et al. (1987) using speckle interferometry at the $4 \mathrm{~m}$ Mayall telescope, without any detection of a secondary companion.

HD 27483.-Flagged by Nordström et al. (2004) as a spectroscopic binary, with a mass ratio of $q=0.937 \pm 0.011$.

HD 27901. - Examined by Mason et al. (1993) using speckle interferometry, without any detection of a secondary companion.

HD 37594.-Found to have a range of spectral classifications from A5 to F2 (Bertaud \& Floquet 1967) to F0 Vp (Abt \&
Morrell 1995) as found by Skiff(2005), indicating that the model fits to these data may be problematic.

HD 43587. - Noted by Nidever et al. (2002) as having a radial velocity rms $>0.1 \mathrm{~km} \mathrm{~s}^{-1}$, and Makarov \& Kaplan (2005) cite the object as a possible astrometric binary from Hipparcos proper motion discrepancies, with the factor $Q_{0}$ as discussed in that study, related to the lower bound of a possible secondary companion's mass, having a value of $\log Q_{0}=-1.6 \mathrm{yr}^{-1}$.

HD 75137.- - Listed by Turon et al. (1993) to have a companion separated by $12.4^{\prime \prime}$ with a magnitude difference of 7.5. Worley \& Douglass (1997) state that the primary in the system is a spectroscopic binary with an orbital period of 8.2 days. It also has high rotational velocity (see below).

HD 79969. - Noted by Horch et al. (2004) using speckle interferometry, to have a secondary companion. The orbital axis is $0.66^{\prime \prime} \pm 0.007^{\prime \prime}$ with a period of $34.2 \mathrm{yr}$ (Henry \& McCarthy 1993).

HD 118232.- Flagged as a suspected variable star by Samus et al. (2004) with the variable type and period unidentified. A visual inspection of the PTI visibility data for the nine nights this object was observed shows seven that are qualitatively identical to the associated calibrators, and two that are marginally offset $\Delta V^{2} \approx 0.05$ from those calibrators.

HD 120510.- Flagged by Nordström et al. (2004) as a spectroscopic binary, with a mass ratio of $q=1.000 \pm 0.009$.

HD 122676.- - Noted by Nidever et al. (2002) as having a radial velocity $\mathrm{rms}>0.1 \mathrm{~km} \mathrm{~s}^{-1}$.

HD 123999.-Flagged by Nordström et al. (2004) as a spectroscopic binary, with a mass ratio of $q=0.963 \pm 0.010$.

HD 141187.- Noted by Makarov \& Kaplan (2005) as a possible astrometric binary from Hipparcos proper motion discrepancies, with $\log Q_{0}=-1.1 \mathrm{yr}^{-1}$.

HD 149630.- Noted by Makarov \& Kaplan (2005) as a possible astrometric binary from Hipparcos proper motion discrepancies, with $\log Q_{0}=-1.0 \mathrm{yr}^{-1}$.

HD 152308.- Noted in Hoffleit \& Jaschek (1991) as having "suspected variable radial velocity." It appears in the GCVS catalog as a rotating variable star (Samus et al. 2004) and has an identified period of 0.9366 days (Renson \& Catalano 2001).

$H D$ 157935.- - Has a radial velocity of $-51.9 \mathrm{~km} \mathrm{~s}^{-1}$ quoted in Duflot et al. (1995; no error given, but a radial velocity quality of " $B$ " on a scale of " $A$ " to " $E$," where " $A$ " is best), but a value of $-54.7 \pm 1.6 \mathrm{~km} \mathrm{~s}^{-1}$ listed in Nordström et al. (2004)possibly an indication of a variable radial velocity.

HD 181655.- Noted by Nidever et al. (2002) as having a stable radial velocity with $\mathrm{rms}<100 \mathrm{~m} \mathrm{~s}^{-1}$.

HD 195050.- Noted by Makarov \& Kaplan (2005) as a possible astrometric binary from Hipparcos proper motion discrepancies, with $\log Q_{0}=-1.1 \mathrm{yr}^{-1}$. It is also seen to have high rotational velocity (see below).

HD 120048, HD 120509, HD 136643, HD 158063, HD 186547, and HD 234677.- -All listed in the radial velocity study of Famaey et al. (2005) as having "no evidence for radial-velocity variations."

HD 216538.-Flagged by Samus et al. (2004) as a long-period pulsating B star.

HD 216831. - A member of a visual double system with a separation of 49.6" and a magnitude difference of 3.76 (Worley \& Douglass 1997). Further, it was examined by McAlister et al. (1987) using speckle interferometry at the $3.6 \mathrm{~m} \mathrm{CFHT,} \mathrm{without}$ any detection of a secondary companion. Finally, it has anomalously high metallicity compared to solar of $[\mathrm{Fe} / \mathrm{H}]=0.40$ (Cayrel de Strobel et al. 1997).

HD 223346. - Flagged to have anomalously low metallicity compared to solar of $[\mathrm{Fe} / \mathrm{H}]=-0.42$ (Ibukiyama \& Arimoto 2002). 
HD 223421.-Flagged to have anomalously low metallicity compared to solar ranging from $[\mathrm{Fe} / \mathrm{H}]=-0.20$ to -0.35 (see, e.g., Ibukiyama \& Arimoto 2002 and Cayrel de Strobel et al. 1997). It is flagged as a probable spectroscopic binary in a catalog of overluminous F-type stars, although identification was problematic (Griffin \& Suchkov 2003).

In addition, the following stars have rotational velocities $\left(v \sin i\right.$ ) between 100 and $200 \mathrm{~km} \mathrm{~s}^{-1}$ : HD 20677, HD 23850, HD 27322, HD 27901, HD 45542, HD 75137, HD 82621, HD 141187 , HD 141851, HD 144874, HD 178449, HD 195050, HD 204403. The following stars have rotational velocities higher than $200 \mathrm{~km} \mathrm{~s}^{-1}$ : HD 149630 and HD 184606. These data were taken mainly from VizieR catalogs associated with the following papers: Glebocki et al. (2000), Royer et al. (2002), and de Medeiros et al. (2002).

\section{CONCLUSION}

We have examined 8 years of PTI visibility data for candidate single, pointlike stellar calibrator sources. To vet the appropriate sources, we subjected the data to a rigorous statistical evaluation, comparing the sources to a well understood and intensively studied standard PTI calibrator, HD 217014. Of the candidates that satisfied the a priori selection criteria, approximately 350 were found to be "well behaved" in an empirical, statistical sense, with $\sim 140$ being rejected. These vetted calibrator objects represent $\gtrsim 96 \%$ sky coverage for PTI in the declination range $5^{\circ}<\delta<55^{\circ}$ and form a set of well characterized calibrator anchors for future PTI observations, archival studies, and observations at other interferometric facilities.

This research has made use of NASA's Astrophysics Data System. This research has made use of the SIMBAD and VizieR databases, operated at CDS, Strasbourg, France. Photometric data was obtained in part from the General Catalog of Photometric Data (Mermilliod et al. 1997). This publication makes use of data products from the Two Micron All Sky Survey, which is a joint project of the University of Massachusetts and the Infrared Processing and Analysis Center/California Institute of Technology, funded by the National Aeronautics and Space Administration and the National Science Foundation. Science operations with PTI are conducted through the efforts of the PTI Collaboration (http:// msc.caltech.edu/missions/Palomar), and we acknowledge the invaluable contributions of our PTI colleagues. Funding for PTI was provided to the Jet Propulsion Laboratory under its TOPS (Towards Other Planetary Systems), ASEPS (Astronomical Studies of Extrasolar Planetary Systems), and Origins programs and from the JPL Director's Discretionary Fund. Portions of this work were performed at the Jet Propulsion Laboratory, California Institute of Technology, under contract with the National Aeronautics and Space Administration.
Abt, H. A., \& Morrell, N. I. 1995, ApJS, 99, 135

Adelman, S. J. 2001, A\&A, 367, 297

Alcaino, G. 1965, Lowell Obs. Bull., 6, 167

Allen, D. A. 1973, MNRAS, 161, 145

Anthony-Twarog, B. J., \& Twarog, B. A. 1994, AJ, 107, 1577

Appenzeller, I. 1966, Z. Astrophys., 64, 269

Ardeberg, A., Sarg, K., \& Wramdemark, S. 1973, A\&AS, 9, 163

Arellano Ferro, A., Parrao, L., Schuster, W., Gonzalez-Bedolla, S., Peniche, R., \& Pena, J. H. 1990, A\&AS, 83, 225

Argue, A. N. 1963, MNRAS, 125, 557 1966, MNRAS, 133, 475

Arp, H. C. 1958, AJ, 63, 118

Baize, P. 1989, A\&AS, 81, 415

Bakos, G. A. 1968, AJ, 73, 187

Barry, D. C. 1969 , PASP, 81,339

Bartkevicius, A. 1984, Vilnius Astronomijos Observatorijos Biuletenis, 68, 3

Bartkowiak, A., \& Jakimiec, M. 1989, Acta Astron., 39, 85

Beichman, C. A., Neugebauer, G., Habing, H. J., Clegg, P. E., \& Chester, T. J. 1988, Infrared Astronomical Satellite (IRAS) Catalogs and Atlases. Vol. 1: Explanatory Supplement, 1

Bertaud, C., \& Floquet, M. 1967, J. Obs., 50, 425

Bessell, M. S., \& Brett, J. M. 1988, PASP, 100, 1134

Blaauw, A., West, R. M., Bartaya, R. A., \& Tolbert, C. R. 1976, A\&AS, 23, 393

Blanco, C., Marilli, E., \& Catalano, S. 1979, A\&AS, 36, 297

Boden, A. F., Colavita, M. M., van Belle, G. T., \& Shao, M. 1998a, Proc. SPIE, 3350,872

Boden, A. F., Creech-Eakman, M. J., \& Queloz, D. 2000, ApJ, 536, 880

Boden, A. F., \& Lane, B. F. 2001, ApJ, 547, 1071

Boden, A. F., Torres, G., \& Hummel, C. A. 2005, ApJ, 627, 464

Boden, A. F., et al. 1998b, ApJ, 504, L39

1999a, ApJ, 515, 356

$1999 \mathrm{~b}$, ApJ, 527, 360

Bok, B. J., Bok, P. F., \& Miller, E. W. 1972, AJ, 77, 733

Bond, H. E. 1970, ApJS, 22, 117 1980, ApJS, 44, 517

Bordé, P., Coudé du Foresto, V., Chagnon, G., \& Perrin, G. 2002, A\&A, 393, 183

Bouigue, R. 1959, Ann. Obs. Astron. Tolouse, 27, 47

Bouigue, R., Boulon, J., \& Pedoussaut, A. 1961, Ann. Obs. Astron. Tolouse, 28,33

2863, Ann. Obs. Astron. Tolouse, 29, 17
REFERENCES

Breger, M. 1968, AJ, 73, 84 1974, ApJ, 188, 53

Bronkalla, W., \& Notni, P. 1961, Astron. Nachr., 286, 179

Burnichon, M. L., \& Garnier, R. 1976, A\&AS, 24, 89

Cameron, R. C. 1966, Georgetown Obs. Monogr., 21, 0

Cardelli, J. A., Clayton, G. C., \& Mathis, J. S. 1989, ApJ, 345, 245

Carney, B. W. 1978, AJ, 83, 1087

1979, ApJ, 233, 211

1983, AJ, 88, 610

Carney, B. W., \& Aaronson, M. 1979, AJ, 84, 867

Cayrel de Strobel, G., Soubiran, C., Friel, E. D., Ralite, N., \& Francois, P. 1997, A\&AS, 124, 299

Celis, S., L. 1975, A\&AS, 22, 9

Claret, A., \& Hauschildt, P. H. 2003, A\&A, 412, 241

Colavita, M. M. 1999, PASP, 111, 111

Colavita, M. M., et al. 1999, ApJ, 510, 505

Coleman, L. A. 1982, AJ, 87, 369

Cousins, A. W. J. 1962a, Mon. Notes Astron. Soc. South Africa, 21, 20

1962b, Mon. Notes Astron. Soc. South Africa, 21, 61 1963a, Mon. Notes Astron. Soc. South Africa, 22, 12 1963b, Mon. Notes Astron. Soc. South Africa, 22, 58 1963c, Mon. Notes Astron. Soc. South Africa, 22, 130 1964a, Mon. Notes Astron. Soc. South Africa, 23, 10 1964b, Mon. Notes Astron. Soc. South Africa, 23, 175 1965, Mon. Notes Astron. Soc. South Africa, 24, 120 1973, Mon. Notes Astron. Soc. South Africa, 32, 117 1984, S. African Astron. Obs. Circ., 8, 59 1987, S. African Astron. Obs. Circ., 11, 93

Cousins, A. W. J., \& Stoy, R. H. 1962, R. Greenwich Obs. Bull., 64, 103

Cowley, A. P., Hiltner, W. A., \& Witt, A. N. 1967, AJ, 72, 1334

Cox, A. N. 2000, Allen's Astrophysical Quantities, ed. Cox, A. N. (4th ed.; New York: AIP)

Crawford, D. L. 1963, ApJ, 137, 523

. 1975, AJ, 80, 955

Crawford, D. L., \& Barnes, J. V. 1969, AJ, 74, 818 1970, AJ, 75, 978 1974, AJ, 79, 687

Crawford, D. L., Barnes, J. V., Faure, B. Q., \& Golson, J. C. 1966, AJ, 71, 709 Crawford, D. L., Barnes, J. V., Gibson, J., Golson, J. C., Perry, C. L., \& Crawford, M. L. 1972, A\&AS, 5, 109

Crawford, D. L., Barnes, J. V., \& Golson, J. C. 1971, AJ, 76, 1058

Crawford, D. L., Barnes, J. V., Golson, J. C., \& Hube, D. P. 1973, AJ, 78, 738 
Crawford, D. L., \& Perry, C. L. 1966, AJ, 71, 206 1976, AJ, 81, 419

Danziger, I. J., \& Dickens, R. J. 1967, ApJ, 149, 55

Danziger, I. J., \& Faber, S. M. 1972, A\&A, 18, 428

Davis, J., Tango, W. J., \& Booth, A. J. 2000, MNRAS, 318, 387

de Medeiros, J. R., Udry, S., Burki, G., \& Mayor, M. 2002, A\&A, 395, 97

de Vaucouleurs, G. 1959, Planet. Space Sci., 2, 26

Deutschman, W. A., Davis, R. J., \& Schild, R. E. 1976, ApJS, 30, 97

Dickens, R. J., Kraft, R. P., \& Krzeminski, W. 1968, AJ, 73, 6

Duflot, M., Figon, P., \& Meyssonnier, N. 1995, A\&AS, 114, 269

Duquennoy, A., \& Mayor, M. 1991, A\&A, 248, 485

Dyck, H. M., Benson, J. A., van Belle, G. T., \& Ridgway, S. T. 1996, AJ, 111, 1705

Echevarria, J., Roth, M., \& Warman, J. 1979, Rev. Mex. AA, 4, 287

Eggen, O. J. 1963, AJ, 68, 483 . 1965, AJ, 70, 19

1966, R. Greenwich Obs. Bull., 120, 333

1971, PASP, 83, 741

1972, ApJ, 175, 787

1973, PASP, 85, 542

Elias, J., Frogel, J., Hyland, A., \& Jones, T. 1983, AJ, 88, 1027

Elias, J., Frogel, J., Matthews, K., \& Neugebauer, G. 1982, AJ, 87, 1029

Elliott, J. E. 1974, AJ, 79, 1082

Epps, E. A. 1972, R. Greenwich Obs. Bull., 176, 127

Erro, B. I. 1969, Boletin del Instituto de Tonantzintla, 5, 89

Evans, D. S. 1966, R. Greenwich Obs. Bull., 110, 185

Fabregat, J., \& Reglero, V. 1990, A\&AS, 82, 531

Fabricius, C., et al. 2002, A\&A, 384, 180

Famaey, B., Jorissen, A., Luri, X., Mayor, M., Udry, S., Dejonghe, H., \& Turon, C. 2005, A\&A, 430, 165

Fernie, J. D. 1969, JRASC, 63, 133 1976, PASP, 88, 116 1983, ApJS, 52, 7

Fernie, J. D., Hagen, J. P. J., Hagen, G. L., \& McClure, L. 1971, PASP, 83, 79

Fischer, D. A., \& Valenti, J. 2005, ApJ, 622, 1102

Fitch, W. S. 1955, ApJ, 121, 690

Franco, G. A. P. 1989, A\&AS, 80, 127

Galland, F., Lagrange, A.-M., Udry, S., Chelli, A., Pepe, F., Queloz, D., Beuzit,

J.-L., \& Mayor, M. 2005, A\&A, 443, 337

Garmany, C. D., \& Ianna, P. A. 1977, A\&AS, 28, 295

Gascoigne, S. C. B. 1962, MNRAS, 124, 201

Gehrels, T., \& Owings, D. 1962, ApJ, 135, 906

Gerbaldi, M., Faraggiana, R., \& Lai, O. 2003, A\&A, 412, 447

Gezari, D. Y., Pitts, P. S., \& Schmitz, M. 1996, Catalog of Infrared Observations, ed. 3.5

Giclas, H. L. 1954, AJ, 59, 128

Giclas, H. L., Burnham, R., \& Thomas, N. G. 1971, Lowell Proper Motion Survey Northern Hemisphere (Flagstaff: Lowell Observatory)

Glass, I. S. 1974, Mon. Notes Astron. Soc. South Africa, 33, 53 1975, MNRAS, 171, 19P

Glebocki, R., Gnacinski, P., \& Stawikowski, A. 2000, Acta Astron., 50, 509

Goldberg, D., Mazeh, T., \& Latham, D. W. 2003, ApJ, 591, 397

Grant, G. 1959, ApJ, 129, 62

Gray, R. O., \& Olsen, E. H. 1991, A\&AS, 87, 541

Griffin, R. F., \& Suchkov, A. A. 2003, ApJS, 147, 103

Gronbech, B., \& Olsen, E. H. 1976, A\&AS, 25, 213

Gronbech, B., Olsen, E. H., \& Stromgren, B. 1976, A\&AS, 26, 155

Guetter, H. H. 1980, PASP, 92, 215

Guetter, H. H., \& Hewitt, A. V. 1984, PASP, 96, 441

Gutierrez-Moreno, A., et al. 1966, Publ. Department of Astronomy University of Chile, 1,1

Häggkvist, L. 1966, Ark. Astron., 4, 165

Häggkvist, L., \& Oja, T. 1966, Ark. Astron., 4, 137 1969a, Ark. Astron., 5, 125

1969b, Ark. Astron., 5, 303 1973, A\&AS, 12, 381

Hale, A. 1994, AJ, 107, 306

Hardie, R. 1958, ApJ, 127, 620

Hardie, R. H., Seyfert, C. K., \& Gulledge, I. S. 1960, ApJ, 132, 361

Harmanec, P., Horn, J., Koubsky, P., Zdarsky, F., Kriz, S., \& Pavlovski, K. 1980, Bull. Astron. Inst. Czechoslovakia, 31, 144

Harris, D. L. 1955, ApJ, 121, 554 1956, ApJ, 123, 371

Harris, D. L., \& Upgren, A. R. 1964, ApJ, 140, 151

Hauck, B., \& Mermilliod, M. 1998, A\&AS, 129, 431

Haug, U. 1970, A\&AS, 1, 35

Haug, U., \& Walter, K. 1970, A\&AS, 1, 29
Haupt, H. F., \& Schroll, A. 1974, A\&AS, 15, 311

Heck, A., \& Manfroid, J. 1975, A\&AS, 22, 323

1980, A\&AS, 42, 311

Heintz, W. D. 1994, AJ, 108, 2338

Henden, A. A. 1980, MNRAS, 192, 621

Henry, T. J., \& McCarthy, D. W., Jr. 1993, AJ, 106, 773

Hilditch, R. W., Hill, G., \& Barnes, J. V. 1976, Mem. Roy. Astron. Soc., 82, 95

Hill, G., \& Barnes, J. V. 1982, Publ. Dominion Astrophys. Obs. Victoria, 16, 71

Hiltner, W. A. 1956, ApJS, 2, 389

Hiltner, W. A., \& Johnson, H. L. 1956, ApJ, 124, 367

Hoag, A. A., Johnson, H. L., Iriarte, B., Mitchell, R. I., Hallam, K. L., \&

Sharpless, S. 1961, Publ. US Naval Observatory Second Series, 17, 343

Hoffleit, D., \& Jaschek, C., ed. 1991, The Bright star catalogue (5th ed.; New

Haven: Yale Univ. Observatory)

Hogg, A. R. 1958, Mount Stromlo Observatory Mimeographs, 2, 1

Horch, E. P., Meyer, R. D., \& van Altena, W. F. 2004, AJ, 127, 1727

Hummel, C. A., Armstrong, J. T., Buscher, D. F., Mozurkewich, D., Quirrenbach, A., \& Vivekanand, M. 1995, AJ, 110, 376

Hummel, C. A., Carquillat, J.-M., Ginestet, N., Griffin, R. F., Boden, A. F., Hajian, A. R., Mozurkewich, D., \& Nordgren, T. E. 2001, AJ, 121, 1623 Ibukiyama, A., \& Arimoto, N. 2002, A\&A, 394, 927

Iriarte, B. 1969, Boletin de los Observatorios Tonantzintla y Tacubaya, 5, 89 1970, Boletin de los Observatorios Tonantzintla y Tacubaya, 5, 317

Iriarte, B., Johnson, H. L., Mitchell, R. I., \& Wisniewski, W. K. 1965, S\&T, 30, 21 Irwin, J. B. 1961, ApJS, 6, 253

Jennens, P. A., \& Helfer, H. L. 1975, MNRAS, 172, 667

Jerzykiewicz, M. 1971, Acta Astron., 21, 501

Jerzykiewicz, M., \& Serkowski, K. 1966, PASP, 78, 546

Johansen, K. T., \& Gyldenkerne, K. 1970, A\&AS, 1, 165

Johnson, H. L. 1952, ApJ, 116, 640

- 1953, ApJ, 117, 361

1955, Ann. d'Astrophys., 18, 292

1964, Boletin de los Observatorios Tonantzintla y Tacubaya, 3, 305

1965a, ApJ, 141, 923

1965b, Comm. Lunar Planet. Lab., 3, 73

1968, in Stars and Stellar Systems, 7, Nebulae and Interstellar Matter,

ed. B. M. Middlehurst \& L. H. Aller (Chicago: Univ. Chicago Press), chap. 5 Johnson, H. L., \& Harris, D. L. 1954, ApJ, 120, 196

Johnson, H. L., Iriarte, B., Mitchell, R. I., \& Wisniewskj, W. Z. 1966, Comm.

Lunar Planet. Lab., 4, 99

Johnson, H. L., \& Knuckles, C. F. 1955, ApJ, 122, 209

-.1957, ApJ, 126, 113

Johnson, H. L., MacArthur, J. W., \& Mitchell, R. I. 1968, ApJ, 152, 465

Johnson, H. L., \& Mitchell, R. I. 1958, ApJ, 128, 31

Johnson, H. L., \& Morgan, W. W. 1953, ApJ, 117, 313

Joner, M. D., Taylor, B. J., Powell, J. M., \& Johnson, S. B. 1995, PASP, 107, 27

Jones, D. H. P. 1969, Acta Astron., 19, 53

Jordi, C., Figueras, F., Torra, J., \& Asiain, R. 1996, A\&AS, 115, 401

Kharchenko, N. V., Piskunov, A. E., \& Scholz, R.-D. 2004, Astron. Nachr., 325,439

Kilkenny, D. 1987, MNRAS, 228, 713

Kilkenny, D., \& Malcolm, G. 1984, MNRAS, 209, 169

Kilkenny, D., \& Menzies, J. W. 1986, MNRAS, 222, 373

Knude, J. K. 1977, A\&AS, 30, 297 1981, A\&AS, 44, 225

Koch, R. H. 1960, AJ, 65, 127

Koen, C., \& Eyer, L. 2002, MNRAS, 331, 45

Konacki, M., \& Lane, B. F. 2004, ApJ, 610, 443

Koresko, C. D., et al. 1998, ApJ, 509, L45

Kornilov, V. G., et al. 1991, Trudy Gosudarstvennogo Astronomicheskogo Instituta, 63, 4

Kozok, J. R. 1985, A\&AS, 61, 387

Kubiak, M. 1973, Acta Astron., 23, 23

Kubinec, W. R. 1973, Publ. Warner Swasey Obs., 1, 1

Lake, R. 1962a, Mon. Notes Astron. Soc. South Africa, 21, 56 1962b, Mon. Notes Astron. Soc. South Africa, 21, 191 1964a, Mon. Notes Astron. Soc. South Africa, 23, 14 1964b, Mon. Notes Astron. Soc. South Africa, 23, 136 1965, Mon. Notes Astron. Soc. South Africa, 24, 41

Landolt, A. U. 1967, AJ, 72, 1012

Latham, D. W., et al. 2002, AJ, 124, 1144

Lee, T. A. 1968, ApJ, 152, 913

Lindgren, H., \& Bernoulli, K. 1980, A\&AS, 42, 335

Ljunggren, B. 1966, Ark. Astron., 3, 535

Ljunggren, B., \& Oja, T. 1961, Uppsala Astron. Obs. Ann., 4, 1 1965, Ark. Astron., 3, 439

Loden, K., Lindblad, P. O., Schober, J., \& Urban, A. 1980, A\&AS, 41, 85 
Lu, P. K., Demarque, P., van Altena, W., McAlister, H., \& Hartkopf, W. 1987, AJ, 94, 1318

Lucke, P. B. 1974, ApJS, 28, 73

Lutz, T. E. 1971, PASP, 83,488

Lutz, T. E., \& Lutz, J. H. 1977, AJ, 82, 431

Makarov, V. V., \& Kaplan, G. H. 2005, AJ, 129, 2420

Malmquist, K. G., Ljunggren, B., \& Oja, T. 1960, Uppsala Astron. Obs. Ann., 4, 1

Manfroid, J., \& Sterken, C. 1987, A\&AS, 71, 539

Marcy, G. W., Butler, R. P., Williams, E., Bildsten, L., Graham, J. R., Ghez, A. M., \& Jernigan, J. G. 1997, ApJ, 481, 926

Markkanen, T. 1977, Observatory and Astrophysics Laboratory University of Helsinki Report, 1 ,

Marlborough, J. M. 1964, AJ, 69, 215

Marsakov, V. A., \& Shevelev, Y. G. 1988, Bull. Cent. Donnees Stellaires, 35, 129 1995, Bull. Cent. Donnees Stellaires, 47, 13

Mason, B. D. 1995, PASP, 107, 299 1997, AJ, 114, 808

Mason, B. D., McAlister, H. A., Hartkopf, W. I., \& Bagnuolo, W. G. 1993, AJ, 105,220

Mason, B. D., et al. 1999, AJ, 117, 1890

Mayor, M., \& Queloz, D. 1995, Nature, 378, 355

Mayor, M., Udry, S., Naef, D., Pepe, F., Queloz, D., Santos, N. C., \& Burnet, M. 2004, A\&A, 415, 391

Mazeh, T., Latham, D. W., \& Stefanik, R. P. 1996, ApJ, 466, 415

McAlister, H. A., Hartkopf, W. I., Hutter, D. J., Shara, M. M., \& Franz, O. G. 1987, AJ, 93, 183

McClure, R. D. 1970, AJ, 75, 41 1976, AJ, 81, 182

McMillan, R. S., Breger, M., Ferland, G. J., \& Loumos, G. L. 1976, PASP, 88, 495 McNamara, B. J. 1976, PASP, 88, 144

Mechler, G. E. 1976, AJ, 81, 107

Mendoza V., E. E. 1958, ApJ, 128, 207

Mendoza, E. E. 1967a, Boletin de los Observatorios Tonantzintla y Tacubaya, 4,106

1967b, Boletin de los Observatorios Tonantzintla y Tacubaya, 4, 149 1969, Boletin de los Observatorios Tonantzintla y Tacubaya, 5, 57

1970, Boletin de los Observatorios Tonantzintla y Tacubaya, 5, 269

Mendoza, E. E., Gomez, V. T., \& Gonzalez, S. 1978, AJ, 83, 606

Mendoza, E. E., \& Gonzalez, S. F. 1974, Rev. Mex. AA, 1, 67

Mendoza, E. E., Rolland, A., \& Rodriguez, E. 1990, A\&AS, 84, 29

Mermilliod, J.-C. 1986, Catalogue of Eggen's UBV Data

Mermilliod, J.-C., Mermilliod, M., \& Hauck, B. 1997, A\&AS, 124, 349

Miczaika, G. R. 1954, AJ, 59, 233

Millis, R. L. 1969, Lowell Obs. Bull., 7, 113

Moffett, T. J., \& Barnes, T. G. 1979, PASP, 91, 180 1980, ApJS, 44, 427

Moreno, H. 1971, A\&A, 12, 442

Mozurkewich, D., et al. 1991, AJ, 101, 2207

Mumford, G. S. 1956, AJ, 61, 213

Nakagiri, M., \& Yamashita, Y. 1979, Ann. Tokyo Astron. Obs., 17, 221

Naur, P. 1955, ApJ, 122, 182

Neckel, H. 1974, A\&AS, 18, 169

Nekrasova, S. V., \& Nikonov, V. B. 1965, Izv. Krymskoj Astrofiz. Obs., 34, 69

Nicolet, B. 1978, A\&AS, 34, 1

Niconov, V. B., Nekrasova, S. V., Polosuina, N. S., Rachkouvsky, N. D., \& Chuvajev, W. K. 1957, Izv. Krymskoj Astrofiz. Obs., 17, 42

Nidever, D. L., Marcy, G. W., Butler, R. P., Fischer, D. A., \& Vogt, S. S. 2002, ApJS, 141, 503

Nikrasova, V., Nikonov, V. B., Polosukhina, N. S., \& Rybka, E. 1962, Izv. Krymskoj Astrofiz. Obs., 27, 228

Nordström, B., et al. 2004, A\&A, 418, 989

Norris, J., Bessell, M. S., \& Pickles, A. J. 1985, ApJS, 58, 463

Oblak, E. 1978, A\&AS, 34, 453 1990, A\&AS, 83, 467

Oja, T. 1963, Ark. Astron., 3, 273 1983, A\&AS, 52, 131 1984, A\&AS, 57, 357

1985a, A\&AS, 59, 461 1985b, A\&AS, 61, 331 1986, A\&AS, 65, 405

Oke, J. B. 1964, ApJ, 140, 689

Olsen, E. H. 1977, A\&AS, 29, 313 1982, A\&AS, 48, 165 1983, A\&AS, 54, 55 1993, A\&AS, 102, 89 1994a, A\&AS, 104, 429 1994b, A\&AS, 106, 257
Olson, E. C. 1974, AJ, 79, 1424

Oosterhoff, P. T. 1960, Bull. Astron. Inst. Netherlands, 15, 199

Pan, X., Shao, M., \& Kulkarni, S. R. 2004, Nature, 427, 326

Parsons, S. B., \& Montemayor, T. J. 1982, ApJS, 49, 175

Pena, J. H., Peniche, R., Mujica, R., Ibanoglu, C., Ertan, A. Y., Tumer, O., Tunca, Z., \& Evren, S. 1993, Rev. Mex. AA, 25, 129

Penprase, B. E. 1992, ApJS, 83, 273

Penston, M. J. 1973, MNRAS, 164, 133

Perry, C. L. 1969a, AJ, 74, 139 1969b, AJ, 74, 705

Perryman, M. A. C., et al. 1997, A\&A, 323, L49

Pfleiderer, J., Dachs, J., \& Haug, U. 1966, Z. Astrophys., 64, 116

Philip, A. G. D., \& Philip, K. 1973, ApJ, 179, 855

Pickles, A. J. 1998, PASP, 110, 863

Piirola, V. 1976, Obs. Astrophys. Laboratory Univ. Helsinki Report, 1, 0

Przybylski, A., \& Kennedy, P. M. 1965, MNRAS, 131, 95

Reglero, V., \& Fabregat, J. 1991, A\&AS, 90, 25

Reglero, V., Gimenez, A., de Castro, E., \& Fernandez-Figueroa, M. J. 1987, A\&AS, 71, 421

Renson, P., \& Catalano, F. A. 2001, A\&A, 378, 113

Richichi, A., \& Percheron, I. 2005, A\&A, 434, 1201

Rodriguez, E., Lopez-Gonzalez, M. J., \& Lopez de Coca, P. 2000, A\&AS, 144, 469

Roman, N. G. 1955, ApJS, 2, 195

Ronen, S., Aragon-Salamanca, A., \& Lahav, O. 1999, MNRAS, 303, 284

Royer, F., et al. 2002, A\&A, 393, 897

Rufener, F. 1976, A\&AS, 26, 275

Rybka, E. 1969, Acta Astron., 19, 229

Samus, N. N., et al. 2004, Combined Catalog of Variable Stars (Moscow: Inst. of Astronomy of Russian Academy of Sciences)

Sandage, A. 1964, ApJ, 139, 442

Santos, N. C., Israelian, G., \& Mayor, M. 2004, A\&A, 415, 1153

Santos, N. C., et al. 2003, A\&A, 398, 363

Schild, R. E. 1973, AJ, 78, 37

Schuster, W. J., \& Nissen, P. E. 1986, Inf. Bull. Variable Stars, 2943, 1

1988, A\&AS, 73, 225

Schuster, W. J., Parrao, L., \& Contreras Martinez, M. E. 1993, A\&AS, 97, 951

Serkowski, K. 1961, Lowell Obs. Bull., 5, 157

Shao, C. Y. 1964, AJ, 69, 858

Sharpless, S. 1952, ApJ, 116, 251 . 1962, ApJ, 136, 767

Shobbrook, R. R., Herbison-Evans, D., Johnston, I. D., \& Lomb, N. R. 1969, MNRAS, 145,131

Sjögren, U. 1964, Ark. Astron., 3, 339

Skiff, B. A. 2005, General Catalogue of Stellar Spectral Classifications (Flagstaff: Lowell Observatory)

Slettebak, A., Bahner, K., \& Stock, J. 1961, ApJ, 134, 195

Snow, T. P., Lamers, H. J. G. L. M., Lindholm, D. M., \& Odelle, A. P. 1994 , ApJS, 95, 163

Sowell, J. R., \& Wilson, J. W. 1993, PASP, 105, 36

Stetson, P. B. 1991, AJ, 102, 589

Stobie, R. S. 1970, MNRAS, 148, 1

Stokes, N. R. 1972a, MNRAS, 159, 165 1972b, MNRAS, 160, 155

Stoy, R. H. 1963, Mon. Notes Astron. Soc. South Africa, 22, 157

Sturch, C. 1972, PASP, 84, 666

Sturch, C. R., \& Helfer, H. L. 1972, AJ, 77, 726

Szabados, L. 1977, Mitt. Sternw. Ungarischen Akad. Wissen. Budapest, 70, 1 . 1981, Commmun. Konkoly Observatory Hungary, 77, 1

Taylor, B. J., \& Joner, M. D. 1992, PASP, 104, 911

Tifft, W. G. 1963, MNRAS, 125, 199

Tokovinin, A. A., \& Smekhov, M. G. 2002, A\&A, 382, 118

Tolbert, C. R. 1964, ApJ, 139, 1105

Torres, G., Boden, A. F., Latham, D. W., Pan, M., \& Stefanik, R. P. 2002, AJ, 124,1716

Turon, C., et al. 1993, Bull. Cent. Donnees Stellaires, 43, 5

van Belle, G. T. 1999, PASP, 111, 1515

van Belle, G. T., \& van Belle, G. 2005, PASP, 117, 1263

van Belle, G. T., et al. 2006, ApJ, 637, 494

van den Bergh, S. 1967, AJ, 72, 70

Veeder, G. J. 1974, AJ, 79, 1056

Walker, R. L. 1971, PASP, 83, 177

Wallerstein, G., Greenstein, J. L., Parker, R., Helfer, H. L., \& Aller, L. H. 1963, ApJ, 137, 280

Wallerstein, G., \& Helfer, H. L. 1961, ApJ, 133, 562

Warren, W. H. 1973, AJ, 78, 192

Warren, W. H., \& Hesser, J. E. 1977, ApJS, 34, 115 
Wesselink, T., van Paradijs, J., Staller, R. F. A., Meurs, E. J. A., \& Kester, D. 1980, Inf. Bull. Variable Stars, 1800, 1

Westerlund, B. E. 1963, MNRAS, 127, 83

Westin, T. N. G. 1982, A\&AS, 49, 561

Wickramasinghe, D. T., \& Warren, P. R. 1976, MNRAS, 177, 59P

Williams, J. A. 1966, AJ, 71, 615

Wolf, S. C., \& Morrison, N. D. 1975, PASP, 87, 231
Wolff, S. C., \& Wolff, R. J. 1971, AJ, 76, 422

Worley, C. E., \& Douglass, G. G. 1997, A\&AS, 125, 523

Wramdemark, S. 1973, A\&AS, 11, 365

Zdanavicius, K., et al. 1972, Vilnius Astronomijos Observatorijos Biuletenis, 34, 3

Zhang, E.-H. 1983, AJ, 88, 825

Zissell, R. 1972, AJ, 77, 610 